\title{
Multi-Stage Robust Chinese Remainder Theorem
}

\author{
Li Xiao, Xiang-Gen Xia, Fellow, IEEE, and Wenjie Wang, Member, IEEE
}

\begin{abstract}
It is well-known that the traditional Chinese remainder theorem (CRT) is not robust in the sense that a small error in a remainder may cause a large reconstruction error. $A$ robust CRT was recently proposed for a special case when the greatest common divisor (ged) of all the moduli is more than 1 and the remaining integers factorized by the ged are co-prime. It basically says that the reconstruction error is upper bounded by the remainder error level $\tau$ if $\tau$ is smaller than a quarter of the ged of all the moduli. In this paper, we consider the robust reconstruction problem for a general set of moduli. We first present a necessary and sufficient condition on the remainder errors with a general set of moduli and also a corresponding robust reconstruction method. This can be thought of as a single-stage robust CRT. We then propose a two-stage robust CRT by grouping the moduli into several groups as follows. First, the single-stage robust CRT is applied to each group. Then, with these robust reconstructions from all the groups, the single-stage robust CRT is applied again across the groups. This is easily generalized to multi-stage robust CRT. With this two-stage robust CRT, the robust reconstruction holds even when the remainder error level $\tau$ is above the quarter of the ged of all the moduli, and an algorithm on how to group a set of moduli for a better reconstruction robustness is proposed in some special cases.
\end{abstract}

Index Terms-Chinese remainder theorem, frequency estimation from undersamplings, greatest common divisor, moduli, robustness.

\section{INTRODUCTION}

$\mathbf{T}$ HE problem of reconstructing a large integer from its several remainders modulo several smaller positive integers (called moduli) may occur in many applications, such as phase unwrapping in radar signal processing [10], [14]-[23] and frequency determination from several undersampled waveforms [8], [9], [12]. The traditional solution for this problem is the Chinese remainder theorem (CRT), see for example, [1], [2], that uniquely formulates the solution from the remainders if all the moduli are co-prime and the large integer is less than the product of all the moduli. When the moduli are not co-prime, the large integer can be uniquely determined if it is less than the least

Manuscript received June 11, 2013; accepted July 11, 2014. Date of publication July 15, 2014; date of current version August 14, 2014. The associate editor coordinating the review of this manuscript and approving it for publication was Dr. Pina Marziliano. The work of L. Xiao and X.-G. Xia was supported in part by the Air Force Office of Scientific Research (AFOSR) under Grant FA9550-12-1-0055. The work of W. Wang was partially supported by the National Natural Science Foundation of China (NSFC) No. 61172092 and the Research Fund for the Doctoral Programs of Higher Education of China No. 20130201110014.

L. Xiao and X.-G. Xia are with the Department of Electrical and Compute Engineering, University of Delaware, Newark, DE 19716 USA (e-mail: lixiao@ee.udel.edu; xxia@ee.udel.edu).

W. Wang is with the School of Electronics and Information Engineering, Xi'an Jiaotong University, Xi'an, 710049, China (e-mail: wjwang@xjtu.edu. cn).

Color versions of one or more figures are available online at ieeexplore.ieee. org.

Digital Object Identifier 10.1109/TSP.2014.2339798 common multiple $(\mathrm{lcm})$ of all the moduli [3], [4], where one may also find the reconstruction methods. However, it is well-known that the above solution is not robust in the sense that a small error in a remainder may cause a large error in the reconstruction solution, which may degrade the performance of its applications in phase unwrapping and frequency determination, since in these applications, signals are usually noisy and the detected remainders may be erroneous. For a fixed set of moduli (this corresponds to a fixed set of sampling rates in the frequency determination problem from undersampled waveforms [8], [9], [12]), the fundamental problems for robustly reconstructing a large integer (or a high frequency) from its erroneous remainders (or the detections from the discrete Fourier transforms of the undersampled waveforms) are: 1) what is the range of such a determinable large integer? 2) how large can the errors in the remainders be for the robustness to hold? Clearly, the larger the answers are in the above two questions, the better a reconstruction algorithm is. For the first question, the answer is usually fixed and the determinable range (called the dynamic range) is the $1 \mathrm{~cm}$ of all the moduli. For the second question, i.e., the robustness, there have been several studies recently [11]-[13]. Robust reconstructions from erroneous remainders were recently proposed in [12], [13] for a special case when the greatest common divisor (gcd) of all the moduli is more than 1 and the remaining integers factorized by the gcd of all the moduli are co-prime. In this special case, a closed-form robust reconstruction from erroneous remainders was proposed in [13] and a necessary and sufficient condition on the remainder errors was also obtained in [13]. It basically says that the reconstruction error is upper bounded by the remainder error level $\tau$ if $\tau$ is smaller than a quarter of the gcd of all the moduli [12]. A special version of this result was obtained earlier in [10]. In some applications, an unknown, such as the phase unwrapping and frequency estimation, is real-valued in general. So, in [13] the closed-form robust CRT algorithm was naturally generalized to real numbers. Also, a lattice based method was proposed in [25] to address the problem of estimating a real unknown distance with a closed-form algorithm using phase measurements taken at multiple co-prime wavelengths. One can see that there are constraints on the moduli in previous works. The constraints on the moduli may, however, limit the robustness when the range, i.e., the dynamic range, of the determinable integers is roughly fixed.

Different from robustly reconstructing a large integer from its erroneous remainders, another existing approach is to accurately determine the large integer by using some of the error-free remainders among all the remainders [5], [6], [9], which may require that significantly many remainders are error-free and a large number of moduli/remainders may be needed. This approach may sacrifice the dynamic range for a given set of moduli (or undersampling rates [9]) and furthermore, in some signal 
processing applications, to obtain error-free remainders may not be even possible, because observed signals are usually noisy. A probabilistic approach to deal with noises in CRT was proposed in [7], where all the moduli are required to be primes.

In this paper, we consider the robust reconstruction problem for a general set of moduli on which the constraint used in [12], [13] is no longer required. We first present a necessary and sufficient condition for the remainder errors for a robust reconstruction from erroneous remainders with a general set of muduli, where a reconstruction method is also proposed. This can be thought of as a single stage robust CRT. We then propose a two-stage robust CRT by grouping the moduli into several smaller groups as follows. First, the single stage robust CRT is applied to each group. Then, with these robust reconstructions from all the groups, the single stage robust CRT is applied again across the groups. Interestingly, with this two-stage robust CRT, the robust reconstruction holds even when the remainder error level $\tau$ is above the quarter of the gcd of all the moduli. The two-stage robust CRT is then easily generalized to multi-stage robust CRT. In this paper, we also propose an algorithm on how to group a set of moduli for a better reconstruction robustness of the two-stage robust CRT in some special cases.

Note that the two-stage robust CRT is first appeared in [24] that is, however, based on the special single stage robust CRT in [13] when the remaining factors of all the moduli after factorizing out their gcd are co-prime. With the two-stage robust CRT obtained in [24], the remainder error level $\tau$ is, in fact, not better than the quarter of the ged of all the moduli. In contrast, our newly proposed two-stage or multi-stage robust CRT in this paper is based on the generalized single stage robust CRT for arbitrary moduli also newly obtained in this paper and as mentioned earlier, the remainder error level $\tau$ can be above the quarter of the ged of all the moduli, i.e., it achieves a better robustness bound than [24] does. We would like to emphasize here that for a general set of moduli, the reconstruction algorithms of integers from their erroneous remainders proposed in this paper have the best known robustness.

This paper is organized as follows. In Section II, we first briefly introduce the robust CRT results obtained in [12], [13]. We then propose our new single stage robust CRT with the necessary and sufficient condition for a general set of moduli. In Section III, we propose two-stage and multi-stage robust CRT. In Section IV, we propose an algorithm on how to group a set of moduli for a better reconstruction robustness of the two-stage robust CRT. In Section V, we present some simulation results on estimating integers with a general set of moduli. In Section VI, we conclude this paper.

\section{Single Stage Robust CRT}

Let us first see the robust remaindering problem. Let $N$ be a positive integer, $0<M_{1}<M_{2}<\cdots<M_{L}$ be $L$ moduli, and $r_{1}, r_{2}, \ldots, r_{L}$ be the $L$ remainders of $N$, i.e.,

$$
N \equiv r_{i} \bmod M_{i} \text { or } N=n_{i} M_{i}+r_{i}
$$

where $0 \leq r_{i}<M_{i}$ and $n_{i}$ is an unknown integer, for $1 \leq$ $i \leq L$. It is not hard to see that $N$ can be uniquely reconstructed from its $L$ remainders $r_{i}$ if and only if $0 \leq N<$
$\operatorname{lcm}\left(M_{1}, M_{2}, \ldots, M_{L}\right)$. If all the moduli $M_{i}$ are co-prime, the CRT provides a simple reconstruction formula [1], [2].

The problem we are interested in this paper is how to robustly reconstruct $N$ when the remainders $r_{i}$ have errors:

$$
0 \leq \tilde{r}_{i} \leq M_{i}-1 \text { and }\left|\tilde{r}_{i}-r_{i}\right| \leq \tau
$$

where $\tau$ is an error level that may be determined by, for example, the signal-to-noise ratio (SNR) and is also called remainder error bound. Now we want to reconstruct $N$ from these erroneous remainders $\tilde{r}_{i}$ and the known moduli $M_{i}$. The basic idea for the robust CRT in the recent studies and also this paper is to accurately determine the unknown integers $n_{i}$ in (1) which are the folding numbers that may cause large errors in the reconstructions if they are erroneous. Therefore, the problem is to accurately determine the folding numbers $n_{i}$ from these erroneous remainders $\tilde{r}_{i}$.

Once $n_{i}$ for $1 \leq i \leq L$ are accurately found, an estimate of $N$ can be given by

$$
\begin{aligned}
\hat{N}(i)=n_{i} M_{i}+\tilde{r}_{i}= & n_{i} M_{i}+r_{i}+\Delta r_{i}, \\
& \text { for any } i, 1 \leq i \leq L,
\end{aligned}
$$

where $\Delta r_{i}=\tilde{r}_{i}-r_{i}$ denote the errors of the remainders. From (2), $\left|\Delta r_{i}\right| \leq \tau$. Then, an estimate of the unknown parameter $N$ is the average of $\hat{N}(i)$ :

$$
\begin{aligned}
\hat{N} & =\left[\frac{1}{L} \sum_{i=1}^{L} \hat{N}(i)\right] \\
& =N+\left[\frac{1}{L} \sum_{i=1}^{L} \Delta r_{i}\right] \\
& =N+\Delta \bar{r}
\end{aligned}
$$

where $\Delta \bar{r}$ is the average of the remainder errors, and [ $\cdot]$ stands for the rounding integer, i.e., for any $x \in \mathbb{R}$ (the set of all reals), $[x]$ is an integer and subject to

$$
-\frac{1}{2} \leq x-[x]<\frac{1}{2}
$$

Clearly in this way the error of the above estimate of $N$ is upper bounded by

$$
|\hat{N}-N| \leq \tau
$$

i.e., $\hat{N}$ is a robust estimate of $N$.

For the above robust remaindering problem, solutions, i.e., robust reconstruction algorithms, have been proposed in [12], [13] for a special case when the ged of all the moduli is more than 1 and the remaining integers factorized by the gcd of all the moduli are co-prime. The main results can be briefly described below.

Let $M$ be the ged of all the moduli $M_{i}$ in (1). Then, $M_{i}=$ $M \Gamma_{i}, 1 \leq i \leq L$, and assume that all $\Gamma_{i}$ for $1 \leq i \leq L$ are co-prime, i.e., the ged of any pair $\Gamma_{i}$ and $\Gamma_{j}$ for $i \neq j$ is 1 . Define $\Gamma \triangleq \Gamma_{1} \Gamma_{2} \ldots \Gamma_{L}$. For $1 \leq i \leq L$, let

$$
\gamma_{i} \triangleq \Gamma_{1} \ldots \Gamma_{i-1} \Gamma_{i+1} \ldots \Gamma_{L}=\Gamma / \Gamma_{i}
$$


where $\gamma_{1} \triangleq \Gamma_{2} \ldots \Gamma_{L}$ and $\gamma_{L} \triangleq \Gamma_{1} \ldots \Gamma_{L-1}$. We now show how to accurately determine the folding numbers $n_{i}$ in [12] and [13], respectively. First, define

$S_{i} \triangleq\left\{\left(\bar{n}_{1}, \bar{n}_{i}\right)=\underset{\substack{\hat{n}_{1}=0,1, \ldots, \gamma_{1}-1 \\ \hat{n}_{i}=0,1, \ldots, \gamma_{i}-1}}{\arg \min }\left|\hat{n}_{i} M_{i}+\tilde{r}_{i}-\hat{n}_{1} M_{1}-\tilde{r}_{1}\right|\right\}$.

Let $S_{i, 1}$ denote the set of all the first components $\bar{n}_{1}$ of the pairs $\left(\bar{n}_{1}, \bar{n}_{i}\right)$ in set $S_{i}$, i.e.,

$$
S_{i, 1} \triangleq\left\{\bar{n}_{1} \mid\left(\bar{n}_{1}, \bar{n}_{i}\right) \in S_{i} \text { for some } \bar{n}_{i}\right\}
$$

and define

$$
S \triangleq \bigcap_{i=2}^{L} S_{i, 1}
$$

It is proved in [12] that if the remainder error bound $\tau$ is smaller than a quarter of $M$, i.e., $\tau<M / 4$, the folding numbers $n_{i}$ for $1 \leq i \leq L$ can be accurately determined from $S$ and $S_{i}$. Set $S$ defined above contains only one element $n_{1}$, and furthermore if $\left(n_{1}, \bar{n}_{i}\right) \in S_{i}$, then $\bar{n}_{i}=n_{i}$. In addition, [12] has proposed a 1-D searching method with the order of $2(L-1) \Gamma_{i}$ searches. When $L$ or $\Gamma_{i}$ gets large, the searching complexity is still high. Then, a closed-form robust CRT algorithm and its necessary and sufficient condition for it to hold have been proposed in [13]. For the closed-form algorithm, we refer the reader to [13] with which the following necessary and sufficient condition for the accurate determination of the folding numbers $n_{i}$ is obtained in [13].

Proposition 1: [13]: Assume that all $\Gamma_{i}$ for $1 \leq i \leq L$ are co-prime and

$$
0 \leq N<\operatorname{lcm}\left(M_{1}, M_{2}, \ldots, M_{L}\right)=M \Gamma_{1} \Gamma_{2} \ldots \Gamma_{L} .
$$

Then, with the closed-form algorithm determining $\hat{n}_{i}$ for $1 \leq$ $i \leq L$ in [13], $\hat{n}_{i}=n_{i}$ for $1 \leq i \leq L$, i.e., the folding numbers $n_{i}$ for $1 \leq i \leq L$ can be accurately determined, if and only if

$$
-M / 2 \leq \Delta r_{i}-\Delta r_{1}<M / 2, \text { for all } 2 \leq i \leq L .
$$

Although the condition (12) in Proposition 1 is necessary and sufficient for the uniqueness of the solution of the folding numbers $n_{i}$, it involves with two remainder errors and is hard to check in practice. However, with this result the following proposition becomes obvious, which coincides with the much simpler sufficient condition in [12].

Proposition 2: [12], [13]: Assume that all $\Gamma_{i}$ for $1 \leq i \leq L$ are co-prime and

$$
0 \leq N<\operatorname{lcm}\left(M_{1}, M_{2}, \ldots, M_{L}\right)=M \Gamma_{1} \Gamma_{2} \ldots \Gamma_{L} .
$$

If the remainder error bound $\tau$ satisfies

$$
\tau<M / 4,
$$

then we have $\hat{n}_{i}=n_{i}$ for $1 \leq i \leq L$, i.e., the folding numbers $n_{i}$ for $1 \leq i \leq L$ can be accurately determined.
As it was mentioned earlier, these robust reconstruction results are based on the assumption that the $\operatorname{gcd} M$ of all the moduli is more than 1 and the remaining integers $\Gamma_{i}$ in the moduli $M_{i}$ factorized by their gcd $M$ are co-prime. For example, $M_{1}=5 \cdot 5=25, M_{2}=5 \cdot 7=35, M_{3}=5 \cdot 16=80$, and $M_{4}=5 \cdot 19=95$, where $M=5$. When the remainder error level $\tau<5 / 4$, any integer less than $5 \cdot 5 \cdot 7 \cdot 16 \cdot 19$ can be reconstructed within the same error level as the remainders from the erroneous remainders by using the algorithms in [12], [13]. A natural question is what will happen if a general set of moduli $M_{i}$ are used. For example, what will happen if $M_{1}=5 \cdot 14=70, M_{2}=5 \cdot 15=75, M_{3}=5 \cdot 16=80$, and $M_{4}=5 \cdot 18=90$ ? First of all, their gcd is $M=5$ and if we divide them by their gcd, we get $\Gamma_{1}=14, \Gamma_{2}=15, \Gamma_{3}=$ $16, \Gamma_{4}=18$ and clearly these four $\Gamma_{i}$ are not co-prime. So, we can not apply the algorithms or results in [12], [13] directly, which may limit the applications in practice.

We next propose an accurate determination algorithm for the folding numbers $n_{i}$ from erroneous remainders for a general set of moduli $M_{i}$ with a new necessary and sufficient condition on the remainder errors. Let us first see an algorithm for $n_{i}$.

Following the algorithm in [13], we can generalize the results as follows. First, from (1) we can equivalently write it as the following system of congruences:

$$
\left\{\begin{array}{c}
N=n_{1} M_{1}+r_{1} \\
N=n_{2} M_{2}+r_{2} \\
\quad \vdots \\
N=n_{L} M_{L}+r_{L} .
\end{array}\right.
$$

We want to determine $n_{i}$ for $1 \leq i \leq L$. To do so, we let the last $L-1$ equations in (15) subtract the first one and we then have

$$
\left\{\begin{array}{l}
n_{1} M_{1}-n_{2} M_{2}=r_{2}-r_{1} \\
n_{1} M_{1}-n_{3} M_{3}=r_{3}-r_{1} \\
\vdots \\
n_{1} M_{1}-n_{L} M_{L}=r_{L}-r_{1} .
\end{array}\right.
$$

Next, denote $m_{1 i}=\operatorname{gcd}\left(M_{1}, M_{i}\right), \Gamma_{1 i}=\frac{M_{1}}{m_{1 i}}, \Gamma_{i 1}=\frac{M_{i}}{m_{1 i}}$, and $q_{i 1}=\frac{r_{i}-r_{1}}{m_{1 i}}$. Then, we can equivalently express (16) again as

$$
\left\{\begin{array}{l}
n_{1} \Gamma_{12}-n_{2} \Gamma_{21}=q_{21} \\
n_{1} \Gamma_{13}-n_{3} \Gamma_{31}=q_{31} \\
\quad \vdots \\
n_{1} \Gamma_{1 L}-n_{L} \Gamma_{L 1}=q_{L 1} .
\end{array}\right.
$$

Since $\Gamma_{1 i}$ and $\Gamma_{i 1}$ are co-prime, by Bézout's lemma (Lemma 1 in [13]) we get

$$
\left\{\begin{array}{l}
n_{1}=q_{i 1} \bar{\Gamma}_{i}+k q_{i 1} \Gamma_{i 1} \\
n_{i}=\frac{q_{i 1}\left(\bar{\Gamma}_{i} \Gamma_{1 i}-1\right)}{\Gamma_{i 1}}+k q_{i 1} \Gamma_{1 i},
\end{array}\right.
$$

where $1 \leq i \leq L, k \in \mathbb{Z}$ (the set of integers) and $\bar{\Gamma}_{i}$ is the modular multiplicative inverse of $\Gamma_{1 i}$ modulo $\Gamma_{i 1}$.

We can use

$$
\hat{q}_{i 1}=\left[\frac{\tilde{r}_{i}-\tilde{r}_{1}}{m_{1 i}}\right]=q_{i 1}+\left[\frac{\Delta r_{i}-\Delta r_{1}}{m_{1 i}}\right]
$$

as an estimate of $q_{i 1}$ for $2 \leq i \leq L$. Recall that [ [ ] stands for the rounding integer which is defined in (5). Let $\hat{n}_{i}$ for $1 \leq i \leq L$ 
be a set of solutions of (17) when $q_{i 1}$ is replaced by $\hat{q}_{i 1}$ for $2 \leq i \leq L$. In summary, we have the following algorithm.

- Step 1: Calculate these values of $m_{1 i}=\operatorname{gcd}\left(M_{1}, M_{i}\right)$, $\Gamma_{1 i}=\frac{M_{1}}{m_{1 i}}$ and $\Gamma_{i 1}=\frac{M_{i}}{m_{1 i}}$ for $2 \leq i \leq L$ from the given moduli $M_{j}$ for $1 \leq j \leq L$, which can be done in advance.

- Step 2: Calculate $\hat{q}_{i 1}$ for $2 \leq i \leq L$ in (19) from the given erroneous remainders $\tilde{r}_{i}$ for $1 \leq i \leq L$.

- Step 3: Calculate the remainders of $\hat{q}_{i 1} \bar{\Gamma}_{i}$ modulo $\Gamma_{i 1}$, i.e.,

$$
\hat{\xi}_{i 1} \equiv \hat{q}_{i 1} \bar{\Gamma}_{i} \bmod \Gamma_{i 1}
$$

for $2 \leq i \leq L$, where $\bar{\Gamma}_{i}$ is the modular multiplicative inverse of $\Gamma_{1 i}$ modulo $\Gamma_{i 1}$ and can be calculated in advance.

- Step 4: Calculate $\hat{n}_{1}$ from the following system of congruences:

$$
\hat{n}_{1} \equiv \hat{\xi}_{i 1} \bmod \Gamma_{i 1}, \quad \text { for } 2 \leq i \leq L,
$$

where moduli $\Gamma_{i 1}$ may not be co-prime, which can be done by using the algorithms in, for example, [3], [4], and especially in [4], a multi-level decoding technique to reconstruct the large integer is proposed.

- Step 5: Calculate $\hat{n}_{i}$ for $2 \leq i \leq L$ :

$$
\hat{n}_{i}=\frac{\hat{n}_{1} \Gamma_{1 i}-\hat{q}_{i 1}}{\Gamma_{i 1}} .
$$

With the above algorithm, we have the following necessary and sufficient condition result for a general set of moduli.

Theorem 1: Let $M_{i}, 1 \leq i \leq L$, be $L$ arbitrarily distinct positive integers as a given set of moduli and $0 \leq N<\operatorname{lcm}\left(M_{1}, M_{2}, \ldots, M_{L}\right)$. Then, $\hat{n}_{i}=n_{i}$ for all $1 \leq i \leq L$, i.e., the folding numbers $n_{i}$ for $1 \leq i \leq L$ can be accurately determined, if and only if for all $2 \leq i \leq L$,

$$
-\operatorname{gcd}\left(M_{1}, M_{i}\right) / 2 \leq \Delta r_{i}-\Delta r_{1}<\operatorname{gcd}\left(M_{1}, M_{i}\right) / 2 .
$$

Proof: We first prove the sufficiency. Considering the condition in (23) and the estimate of $q_{i 1}$ in (19), from (5) for the definition of the operator [ $\cdot]$ we have $\hat{q}_{i 1}=q_{i 1}$. Then, from (18), $n_{1}$ and $\hat{q}_{i 1} \bar{\Gamma}_{i}$ have the same remainder modulo $\Gamma_{i 1}$. Since $\hat{q}_{i 1}, \bar{\Gamma}_{i}$ and $\Gamma_{i 1}$ are known, we can calculate $\hat{q}_{i 1} \bar{\Gamma}_{i} \equiv \hat{\xi}_{i 1} \bmod \Gamma_{i 1}$. Thus, $n_{1} \equiv \hat{\xi}_{i 1} \bmod \Gamma_{i 1}$ for $2 \leq i \leq L$, which form a system of simultaneous congruences as $\hat{n}_{1} \equiv \hat{\xi}_{i 1} \bmod \Gamma_{i 1}$. In addition, since $n_{1} M_{1} \leq N<\operatorname{lcm}\left(M_{1}, M_{2}, \ldots, M_{L}\right)$, it is not hard to see that $n_{1}$ is less than $\operatorname{lcm}\left(\Gamma_{21}, \Gamma_{31}, \ldots, \Gamma_{L 1}\right)$. So, according to the algorithm about generalized CRT in [4], $n_{1}$ can be uniquely reconstructed by solving the above system, and $n_{1}=\hat{n}_{1}$.

After $n_{1}$ is determined, we can obtain other integers $n_{i}$ for $2 \leq i \leq L$ from (17) or (18). Therefore, $\hat{n}_{i}=n_{i}$ for $2 \leq i \leq L$. Hence, the sufficiency is proved.

We next prove the necessity. Assume that there exists at least one remainder that does not satisfy (23). For example, the $j$-th remainder $\tilde{r}_{j}, 2 \leq j \leq L$, does not satisfy (23). This equivalently leads to $\left[\left(\Delta r_{j}-\Delta r_{1}\right) / m_{1 j}\right] \neq 0$ and therefore $\hat{q}_{j 1} \neq q_{j 1}$. We then have the following two cases.

Case $A$ : When $\left[\left(\Delta r_{j}-\Delta r_{1}\right) / m_{1 j}\right] \neq k \Gamma_{j 1}$ for any $k \in$ $\mathbb{Z}$. We want to prove that the remainders of $\hat{q}_{j 1} \bar{\Gamma}_{j}$ and $q_{j 1} \bar{\Gamma}_{j}$ modulo $\Gamma_{j 1}$ are different. Assume $\hat{q}_{j 1} \bar{\Gamma}_{j}$ and $q_{j 1} \bar{\Gamma}_{j}$ have the same remainder modulo $\Gamma_{j 1}$, i.e.,

$$
\hat{q}_{j 1} \bar{\Gamma}_{j}-q_{j 1} \bar{\Gamma}_{j}=k \Gamma_{j 1}, \quad \text { for some } k \in \mathbb{Z}
$$

Multiplying both sides of (24) by $\Gamma_{1 j}$ and considering $\Gamma_{1 j} \bar{\Gamma}_{j}=$ $1+k \Gamma_{j 1}$ for some $k \in \mathbb{Z}$, we have

$$
\hat{q}_{j 1}-q_{j 1}=k \Gamma_{j 1}, \quad \text { for some } k \in \mathbb{Z} .
$$

According to (19), we have

$$
\left[\left(\Delta r_{j}-\Delta r_{1}\right) / m_{1 j}\right]=k \Gamma_{j 1}, \quad \text { for some } k \in \mathbb{Z} \text {. }
$$

This contradicts with the assumption. Hence, the remainders of $\hat{q}_{j 1} \bar{\Gamma}_{j}$ and $q_{j 1} \bar{\Gamma}_{j}$ modulo $\Gamma_{j 1}$ are different, i.e., $n_{1}$ and $\hat{n}_{1}$ have different congruences. Thus, $n_{1} \neq \hat{n}_{1}$.

Case B: For every $2 \leq i \leq L,\left[\left(\Delta r_{i}-\Delta r_{1}\right) / m_{1 i}\right]=k \Gamma_{i 1}$ for some $k \in \mathbb{Z}$ but there exists at least one $j$ with $2 \leq j \leq L$ such that $\left[\left(\Delta r_{j}-\Delta r_{1}\right) / m_{1 j}\right] \neq 0$, i.e., $\hat{q}_{j 1} \neq q_{j 1}$. From (19), we have $\hat{q}_{i 1} \bar{\Gamma}_{i} \equiv q_{i 1} \bar{\Gamma}_{i} \bmod \Gamma_{i 1}$ for $2 \leq i \leq L$. Hence, from the first equation in (18) and according to the generalized CRT, $n_{1}$ can be uniquely reconstructed. Thus, from Steps $1-4$ in the above algorithm, we have $\hat{n}_{1}=n_{1}$.

However, since $\hat{q}_{j 1} \neq q_{j 1}$, from (17) or the second equation in (18) we have $n_{j} \neq \hat{n}_{j}$. This proves the necessity.

The above result involves with two remainder errors and is hard to check in practice. Let $\tau$ be the maximal remainder error level, i.e., $\left|\Delta r_{i}\right|=\left|\tilde{r}_{i}-r_{i}\right| \leq \tau$, for $1 \leq i \leq L$. Similar to Proposition 2, we can also present a simpler sufficient condition.

Corollary 1: Let $M_{i}, 1 \leq i \leq L$, be $L$ arbitrarily distinct positive integers as a given set of moduli and $0 \leq N<\operatorname{lcm}\left(M_{1}, M_{2}, \ldots, M_{L}\right)$. If the remainder error bound $\tau$ satisfies

$$
\tau<\max _{1 \leq i \leq L} \min _{1 \leq j \neq i \leq L} \frac{\operatorname{gcd}\left(M_{i}, M_{j}\right)}{4},
$$

then, we have $\hat{n}_{i}=n_{i}$ for all $1 \leq i \leq L$, i.e., the folding numbers $n_{i}$ for $1 \leq i \leq L$ can be accurately determined.

Proof: Recall that in the procedure of proving Theorem 1 we just arbitrarily selected the first equation in (15) to be a reference to be subtracted from the other equations to get (16). In fact, to improve the robustness through selecting a proper reference equation to differentiate, we can choose the index $i$ such that $\min \operatorname{gcd}\left(M_{i}, M_{j}\right) \geq \min \operatorname{gcd}\left(M_{l}, M_{j}\right)$ for $l \neq i$. Without loss of generality, modulus $M_{1}$ satisfies

$$
\max _{1 \leq i \leq L} \min _{1 \leq j \neq i \leq L} \frac{\operatorname{gcd}\left(M_{i}, M_{j}\right)}{4}=\min _{1 \leq j \neq 1 \leq L} \frac{\operatorname{gcd}\left(M_{1}, M_{j}\right)}{4} .
$$

Then, we have $\tau<\operatorname{gcd}\left(M_{1}, M_{i}\right) / 4$ for $i \neq 1$. Since $\tau$ is the maximal remainder error level, i.e., $\left|\Delta r_{i}\right|=\left|\tilde{r}_{i}-r_{i}\right| \leq \tau$, for $1 \leq i \leq L$, we can obtain

$$
\left|\Delta r_{i}-\Delta r_{1}\right|<\operatorname{gcd}\left(M_{1}, M_{i}\right) / 2, \quad \text { for } 2 \leq i \leq L .
$$

Clearly, (29) implies the sufficient condition (23) in Theorem 1. Hence, $\hat{n}_{i}=n_{i}$ for all $1 \leq i \leq L$. Therefore, we complete the proof.

Remark 1: Since in the above new result, there is no any constraint to the moduli $M_{i}$ for $1 \leq i \leq L$, some of the moduli may be redundant with respect to the range $0 \leq N<$ $\operatorname{lcm}\left(M_{1}, M_{2}, \ldots, M_{L}\right)$ of the determinable unknown integer $N$. The first case is when there exist a pair of moduli $M_{i_{1}}$ and $M_{i_{2}}$ such that $M_{i_{1}}=n M_{i_{2}}$ for $n \in \mathbb{N}$ (the set of all positive 
integers) and in this case $M_{i_{2}}$ is redundant for the determinable range of $N$, i.e., the $1 \mathrm{~cm}$ of all $M_{i}$. The other case is when there exists one moduli $M_{i_{3}}$ that is a factor of some other (more than one) moduli's lcm, i.e., $\operatorname{lcm}\left(M_{i_{4}}, M_{i_{5}}, \ldots, M_{i_{k}}\right)=n M_{i_{3}}$ for some $n \in \mathbb{N}$ and $k>4$, and in this case $M_{i_{3}}$ is redundant similarly. When a determinable range of $N$ is fixed, we can add or delete some of the redundant moduli to or from the moduli set in order to get a better robustness bound for $\tau$. For example, the redundant modulus 30 in moduli set $\{20,45,30\}$ improves the robustness bound compared with the robustness bound of moduli set $\{20,45\}$ from $5 / 4$ to $10 / 4$. On the other hand, the redundant modulus 10 in $\{10,45,30\}$ with its robustness bound $10 / 4$ does not help but worsens the robustness bound compared with $15 / 4$ of $\{45,30\}$, so it is better to delete the modulus 10 from the moduli set. Below is a general result.

Corollary 2: If there exist a pair of moduli $M_{i_{1}}$ and $M_{i_{2}}$ such that $M_{i_{1}}=n M_{i_{2}}$ for $n \in \mathbb{N}$, then, the redundant modulus $M_{i_{2}}$ does not help to increase the robustness bound and it can be deleted from the set of moduli.

Proof: Without loss of generality, we can assume for a moduli set $\left\{M_{1}, M_{2}, \ldots, M_{L}\right\}$ that $\operatorname{gcd}\left(M_{1}, M_{2}\right) \geq$ $\operatorname{gcd}\left(M_{1}, M_{3}\right) \geq \cdots \geq \operatorname{gcd}\left(M_{1}, M_{L}\right)$ and the robustness bound is $\max _{1<i<L} \min _{1<j \neq i<L} \frac{\operatorname{gcd}\left(M_{i}, M_{j}\right)}{4}=\frac{\operatorname{gcd}\left(M_{1}, M_{L}\right)}{4}$.

Consider another set of moduli $\left\{M_{1}, M_{2}, \ldots, M_{L}, M_{L+1}\right\}$ where $M_{L+1}$ is a factor of one moduli $M_{q}$ in $\left\{M_{1}, M_{2}, \ldots, M_{L}\right\}$, i.e., $M_{q}=n M_{L+1}$ for $n \in \mathbb{N}, 1 \leq q \leq L$. For the moduli $\left\{M_{1}, M_{2}, \ldots, M_{L}, M_{L+1}\right\}$, its robustness bound is $\max _{1<i<L+1} \min _{1<j \neq i \leq L+1} \frac{\operatorname{gcd}\left(M_{i}, M_{j}\right)}{4}$.

To calculate it, we split $1 \leq i \leq L+1$ into two parts: $1 \leq$ $i \leq L$ and $i=L+1$, i.e., (30), shown at the bottom of the page.

As for $A$, since

$$
\min _{\substack{1 \leq j \neq i \leq L+1 \\ 1 \leq i \leq L}} \frac{\operatorname{gcd}\left(M_{i}, M_{j}\right)}{4} \leq \min _{\substack{1 \leq j \neq i \leq L \\ 1 \leq i \leq L}} \frac{\operatorname{gcd}\left(M_{i}, M_{j}\right)}{4},
$$

we have

$$
A \leq \max _{1 \leq i \leq L} \min _{1 \leq j \neq i \leq L} \frac{\operatorname{gcd}\left(M_{i}, M_{j}\right)}{4}=\frac{\operatorname{gcd}\left(M_{1}, M_{L}\right)}{4} .
$$

As for $B$, since

$$
\operatorname{gcd}\left(M_{L+1}, M_{j}\right) \leq \operatorname{gcd}\left(M_{q}, M_{j}\right) \text { for } 1 \leq j \leq L,
$$

we have (31), shown at the bottom of the page.

Thus, we can derive (32), shown at the bottom of the page. This tells us that the redundant modulus $M_{L+1}$ does not help to increase the robustness bound of the set of moduli $\left\{M_{1}, M_{2}, \ldots, M_{L}, M_{L+1}\right\}$ compared with that of $\left\{M_{1}, M_{2}, \ldots, M_{L}\right\}$.

From the result of Corollary 2, for a set of moduli, we can delete this kind of redundant modulus $M_{i_{2}}$ when there exists one modulus $M_{i_{1}}$ in the moduli set such that $M_{i_{1}}=n M_{i_{2}}$. So, throughout this paper, a set of moduli we consider does not include such a pair of moduli in a single stage robust CRT.

From the above results, one can see that the choice of the reference remainder is important in determining the maximal possible robustness bound for $\tau$ when the whole moduli set of $L$ arbitrary moduli is considered once as above. In fact, when the moduli satisfy the constraint, i.e., $\Gamma_{i}$ are co-prime, in Proposition 2 in [12], [13], it has been pointed out and analyzed in [13] that a proper reference remainder indeed plays an important role in improving the performance in practice.

Going back to the necessary and sufficient condition (23), one can see that the remainder error difference bound depends on $\operatorname{gcd}\left(M_{1}, M_{i}\right)$, that varies with each $M_{i}$ and the choice of the reference modulus $M_{1}$. This means that for the robust reconstruction of $N$, the error levels of its remainders $\tilde{r}_{i}$ for different $i$ may have different requirements. Also, as it was mentioned earlier, using $M_{1}$ as the reference modulus is not necessary. Let us choose the reference modulus $M_{k}$ that satisfies

$$
\min _{1 \leq j \neq k \leq L} \frac{\operatorname{gcd}\left(M_{k}, M_{j}\right)}{4}=\max _{1 \leq i \leq L} \min _{1 \leq j \neq i \leq L} \frac{\operatorname{gcd}\left(M_{i}, M_{j}\right)}{4}
$$

and the remainder error bound $\tau_{k}$ for the reference remainder $r_{k}$ satisfy

$$
\left|\Delta r_{k}\right|=\left|\tilde{r}_{k}-r_{k}\right| \leq \tau_{k}<\min _{1 \leq j \neq k \leq L} \frac{\operatorname{gcd}\left(M_{k}, M_{j}\right)}{4} .
$$

Then, we have the following result.

Corollary 3: Let $M_{i}, 1 \leq i \leq L$, be $L$ arbitrarily distinct positive integers as a given set of moduli and $0 \leq N<$ $\operatorname{lcm}\left(M_{1}, M_{2}, \ldots, M_{L}\right)$, define the remainder error bound for $r_{i}$ as $\tau_{i}$, i.e., $\left|\Delta r_{i}\right|=\left|\tilde{r}_{i}-r_{i}\right| \leq \tau_{i}$ for $1 \leq i \leq L$, and the reference modulus and its corresponding remainder error bound are $M_{k}$ and $\tau_{k}$ satisfying (33) and (34) above for some $k$ with $1 \leq k \leq L$. If the remainder error bound $\tau_{i}, 1 \leq i \neq k \leq L$, satisfies

$$
\begin{aligned}
\left|\Delta r_{i}\right| & =\left|\tilde{r}_{i}-r_{i}\right| \leq \tau_{i} \\
& \leq \frac{\operatorname{gcd}\left(M_{k}, M_{i}\right)}{2}-\min _{1 \leq j \neq k \leq L} \frac{\operatorname{gcd}\left(M_{k}, M_{j}\right)}{4},
\end{aligned}
$$

then, we have $\hat{n}_{i}=n_{i}$ for all $1 \leq i \leq L$, i.e., the folding numbers $n_{i}$ for $1 \leq i \leq L$ can be accurately determined.

$$
\begin{aligned}
& \max _{1 \leq i \leq L+1} \min _{1 \leq j \neq i \leq L+1} \frac{\operatorname{gcd}\left(M_{i}, M_{j}\right)}{4}=\max \{\underbrace{\max _{1 \leq j \neq i \leq L+1} \min _{A} \frac{\operatorname{gcd}\left(M_{i}, M_{j}\right)}{4}, \underbrace{\min _{1 \leq L} \frac{\operatorname{gcd}\left(M_{L+1}, M_{j}\right)}{4}}_{1 \leq j \leq L}\} .}_{1 \leq i \leq L} \\
& B \leq \min _{1 \leq j \neq q \leq L} \frac{\operatorname{gcd}\left(M_{L+1}, M_{j}\right)}{4} \leq \max _{1 \leq i \leq L} \min _{1 \leq j \neq i \leq L} \frac{\operatorname{gcd}\left(M_{i}, M_{j}\right)}{4}=\frac{\operatorname{gcd}\left(M_{1}, M_{L}\right)}{4} . \\
& \max _{1 \leq i \leq L+1} \min _{1 \leq j \neq i \leq L+1} \frac{\operatorname{gcd}\left(M_{i}, M_{j}\right)}{4}=\max \{A, B\} \leq \max _{1 \leq i \leq L} \min _{1 \leq j \neq i \leq L} \frac{\operatorname{gcd}\left(M_{i}, M_{j}\right)}{4}=\frac{\operatorname{gcd}\left(M_{1}, M_{L}\right)}{4} .
\end{aligned}
$$


Proof: If the reference modulus and its corresponding remainder error bound are $M_{k}$ and $\tau_{k}, 1 \leq k \leq L$, we just need to prove $\left|\Delta r_{i}-\Delta r_{k}\right|<\operatorname{gcd}\left(M_{i}, M_{k}\right) / 2$ for all $1 \leq i \neq k \leq L$, which implies the sufficient condition (23) in Theorem 1.

have

Since $\left|\Delta r_{i}\right| \leq \tau_{i} \leq \frac{\operatorname{gcd}\left(M_{k}, M_{i}\right)}{2}-\min _{1 \leq j \neq k \leq L} \frac{\operatorname{gcd}\left(M_{k}, M_{j}\right)}{4}$, we

$$
\begin{aligned}
\left|\Delta r_{i}-\Delta r_{k}\right| & \leq\left|\Delta r_{i}\right|+\left|\Delta r_{k}\right| \\
& \leq \tau_{i}+\tau_{k}<\frac{\operatorname{gcd}\left(M_{k}, M_{i}\right)}{2} .
\end{aligned}
$$

Thus, Corollary 3 is proved.

Next, we consider the example mentioned before again.

Example 1: Let $M_{1}=5 \cdot 14=70, M_{2}=5 \cdot 15=75, M_{3}=$ $5 \cdot 16=80$, and $M_{4}=5 \cdot 18=90$. It is easy to see that their gcd is $M=5$, and $\left\{M_{i}\right\}_{i=1,2,3,4}$ do not satisfy the constraint of Propositions 1 and 2 in [12], [13]. Thus, their results can not be applied here. However, from the result of Corollary 1 , we can obtain the maximal robustness bound $\tau$ for all remainders as $10 / 4$, which is even larger than $5 / 4$, a quarter of the gcd of all the moduli. From the result of Corollary 3, we choose $M_{4}$ as the reference modulus that does satisfy (33), and we can get the robustness bound for each remainder as follows: $\tau_{1} \leq 10 / 4, \tau_{2} \leq 20 / 4, \tau_{3} \leq 10 / 4$ and $\tau_{4}<10 / 4$. One can see that, if we treat remainder error bounds individually as above, the remainder error bounds for some of the individual remainders, such as the second remainder in this example, may be larger than that in (27) in Corollary 1 for all the remainder error levels. In addition, the robust reconstruction range of $N$ is also $0 \leq N<\operatorname{lcm}\left(M_{1}, M_{2}, M_{3}, M_{4}\right)$.

It is clear that when moduli $\Gamma_{i 1}$ for $2 \leq i \leq L$ are co-prime similar to the case of [13], from the system of congruences (21) in Step 4 in the above algorithm, a closed-form single stage robust CRT can be obtained as [13] and we can replace Step 4 with the following Step $4^{\star}$.

- Step $4^{\star}$ : Calculate $\hat{n}_{1}$ :

$$
\hat{n}_{1}=\sum_{i=2}^{L} \hat{\xi}_{i 1} b_{i 1} \frac{\gamma}{\Gamma_{i 1}} \bmod \gamma,
$$

where $b_{i 1}$ is the modular multiplicative inverse of $\gamma / \Gamma_{i 1}$ modulo $\Gamma_{i 1}$, which can be calculated in advance, and $\gamma=\Gamma_{21} \Gamma_{31} \ldots \Gamma_{L 1}$. After that, from (22) we can get the formulas for other $\hat{n}_{i}$ for $2 \leq i \leq L$.

Next, let us consider the result in [24]. If we consider the following special case of moduli in Corollary 3 , we can obtain a better result of the remainder error bounds than that in [24]. Let a set of moduli be $\left\{M_{1}, M_{2}, \ldots, M_{L_{1}+L_{2}}\right\}=$ $\left\{M \Gamma_{1,1}, \ldots, M \Gamma_{1, L_{1}}, M M^{\prime} \Gamma_{2,1}, \ldots, M M^{\prime} \Gamma_{2, L_{2}}\right\}$, where $L_{1} \geq 2$. Then, we have the following corollary.

Corollary 4: Assume that all the $\Gamma_{j, i}, 1 \leq i \leq L_{j}, j=1,2$, are co-prime, let $\Gamma_{1,1}=\dot{\Gamma}_{1,1} M^{\prime}$, where $\dot{\Gamma}_{1,1}$ is an integer, and $0 \leq N<\operatorname{lcm}\left(M_{1}, M_{2}, \ldots, M_{L_{1}+L_{2}}\right)$. Denote $\tau_{i}$ as the error bound for each remainder $r_{i}$ for $1 \leq i \leq L_{1}+L_{2}$. If

$$
\begin{aligned}
&\left|\Delta r_{1}\right| \leq \tau_{1}<M / 4, \\
&\left|\Delta r_{i}\right| \leq \tau_{i} \leq M / 4, \quad \text { for all } 2 \leq i \leq L_{1}, \\
&\left|\Delta r_{i}\right| \leq \tau_{i} \leq M M^{\prime} / 2-M / 4 \\
& \quad \text { for all } L_{1}+1 \leq i \leq L_{1}+L_{2},
\end{aligned}
$$

then, with a closed-form algorithm we have $\hat{n}_{i}=n_{i}$ for $1 \leq$ $i \leq L_{1}+L_{2}$, i.e., the folding numbers $n_{i}$ for $1 \leq i \leq L_{1}+L_{2}$ can be accurately determined.

Proof: Since $\min _{1<j \leq L_{1}+L_{2}} \frac{\operatorname{gcd}\left(M_{1}, M_{j}\right)}{4}=\max _{1 \leq i \leq L_{1}+L_{2}}$ $\min _{j \neq i \leq L_{1}+L_{2}} \frac{\operatorname{gcd}\left(M_{i}, M_{j}\right)}{4}$, we can set $M_{1}$ as the reference modulus and the error bound $\tau_{1}<\min _{1<j \leq L_{1}+L_{2}} \frac{\operatorname{gcd}\left(M_{1}, M_{j}\right)}{4}=\frac{M}{4}$ in (34).

Then, from (35) when $2 \leq i \leq L_{1}, \tau_{i} \leq M / 2-M / 4=$ $M / 4$; and when $L_{1}+1 \leq i \leq L_{1}+L_{2}, \tau_{i}<M M^{\prime} / 2-M / 4$. So we can accurately determine the folding numbers $n_{i}$ for $1 \leq$ $i \leq L_{1}+L_{2}$. Next, we can get $\Gamma_{i 1}=\frac{M_{i}}{\operatorname{gcd}\left(M_{1}, M_{i}\right)}=\Gamma_{1, i}$ for $2 \leq i \leq L_{1}$ and $\Gamma_{i 1}=\Gamma_{2, i}$ for $L_{1}+1 \leq i \leq L_{1}+L_{2}$, all of which are co-prime. Thus, we can obtain a simple closed-form reconstruction formula for $\hat{n}_{1}$ similar to (37) and then $\hat{n}_{i}$ by (22) for $2 \leq i \leq L_{1}+L_{2}$.

Example 2: In the above, let $M=10, M^{\prime}=3, \Gamma_{1.1}=33$, $\Gamma_{1,2}=31, \Gamma_{2,1}=35, \Gamma_{2,2}=37$, and the moduli are $\left\{M_{1}, M_{2}, M_{3}, M_{4}\right\}=\{10 \cdot 33,10 \cdot 31,30 \cdot 35,30 \cdot 37\}$. From Corollary 4 , we can get $\tau_{1}<10 / 4, \tau_{2} \leq 10 / 4, \tau_{3} \leq$ $50 / 4, \tau_{4} \leq 50 / 4$. In addition, it has a closed-form algorithm to robustly reconstruct an unknown integer $N$ for $0 \leq N<\operatorname{lcm}\left(M_{1}, M_{2}, M_{3}, M_{4}\right)$. However, according to the result of [24], the remainder error bounds would be $\tau_{1}<10 / 4, \tau_{2}<10 / 4, \tau_{3}<30 / 4, \tau_{4}<30 / 4$.

Interestingly, the robustness bound result in this corollary is even better than that obtained in [24] using a two-stage robust CRT. What the result here tells us that for the set of moduli in Corollary 4, which is the set considered in [24], it is not necessary to use a two-stage robust CRT as what is done in [24]. Another remark we make here is that the notation $\tau_{i}$ above denotes the $i$-th remainder error bound. Later, without causing any notational confusion, $\tau_{j}$ will denote the remainder error bound for the remainders in the $j$-th group.

\section{Multi-Stage Robust CRT}

From the study in the previous section, one can see that the robustness bound is kind of dependent on the gcd of the moduli. The larger the gcd is, the better the robustness bound is. However, the large gcd reduces the $1 \mathrm{~cm}$ of the moduli, i.e., reduces the determinable range of the unknown integer $N$. When a set of moduli are given, the maximal determinable range is given too, which is their $1 \mathrm{~cm}$. Then, the question is for a given set of moduli, can we improve the robustness bound obtained in Corollary 1 ? Note that in the single stage robust CRT obtained in the previous section, all the remainders and their related system of congruence equations are considered and solved together simultaneously. A natural question is: can we split the set of moduli into several groups so that the moduli in each group have a large gcd and remainders and their corresponding system of congruence equations in each group are considered and solved independently using the single stage robust CRT obtained in the previous section? If so, can we obtain a better robustness bound than that in Corollary 1 for the single stage robust CRT? To answer these questions, let us first see an example.

Suppose that a set of moduli $\{180,220,486,513\}$ are given and the gcd of these 4 moduli is 1 . These four moduli can be split to two groups $\{180,220\}$ and $\{486,513\}$. The ged of the 
two moduli in the first group is $M=20$ with $\Gamma_{1}=180 / 20=$ $9, \Gamma_{2}=220 / 20=11$, and the gcd of the two moduli in the second group is $M=27$ with $\Gamma_{1}=486 / 27=18, \Gamma_{2}=$ $513 / 27=19$. One can see that each group satisfies the condition in Propositions 1 and 2 and therefore the closed-form robust CRT in [13] or the single stage robust CRT in the previous section can be applied for the robust reconstruction of an unknown integer $N$ with robustness bound $\tau<20 / 4$ or $\tau<27 / 4$, if $N<\operatorname{lcm}(180,220)=1980 \triangleq q_{1}=18 \cdot 110$ or $N<\operatorname{lcm}(486,513)=9234 \triangleq q_{2}=18 \cdot 513$, respectively.

Using the first group with moduli $M_{1}=180$ and $M_{2}=220$ and two remainders $r_{1}$ and $r_{2}$, if the integer $N$ is in the range of $\left[0, \operatorname{lcm}\left(M_{1}, M_{2}\right)\right)$, then $N$ can be uniquely determined by its two error free remainders $r_{1}$ and $r_{2}$ as $N_{1}$ with $0 \leq N_{1}<q_{1}$; otherwise

$$
N=N_{1} \bmod q_{1}
$$

Using two erroneous remainders $\tilde{r}_{1}$ and $\tilde{r}_{2}$ with error level $\tau$, and the closed-form robust CRT in [13] or the single stage robust CRT in the previous section for the first group, we can obtain an integer $\hat{N}_{1}$ and

$$
\text { if } \tau<\operatorname{gcd}\left(M_{1}, M_{2}\right) / 4=20 / 4 \text {, then }\left|N_{1}-\hat{N}_{1}\right| \leq \tau \text {. }
$$

Similarly, using the second group with moduli $M_{3}=486$ and $M_{4}=513$ and two remainders $r_{3}$ and $r_{4}$, if the integer $N$ is in the range of $\left[0, \operatorname{lcm}\left(M_{3}, M_{4}\right)\right)$, then $N$ can be uniquely determined by its two error free remainders $r_{3}$ and $r_{4}$ as $N_{2}$ with $0 \leq N_{2}<q_{2}$; otherwise

$$
N=N_{2} \bmod q_{2}
$$

Using two erroneous remainders $\tilde{r}_{3}$ and $\tilde{r}_{4}$ with error level $\tau$, and the closed-form robust CRT in [13] or the single stage robust CRT in the previous section for the second group, we can obtain an integer $\hat{N}_{2}$ and

$$
\text { if } \tau<\operatorname{gcd}\left(M_{3}, M_{4}\right) / 4=27 / 4 \text {, then }\left|N_{2}-\hat{N}_{2}\right| \leq \tau \text {. }
$$

On the other hand, if integer $N$ is in the range of $\left[0, \operatorname{lcm}\left(M_{1}, M_{2}, M_{3}, M_{4}\right)\right)=\left[0, \operatorname{lcm}\left(q_{1}, q_{2}\right)\right)$, it can be uniquely determined by its four error free remainders $r_{1}, r_{2}, r_{3}, r_{4}$. This can be done either from the four remainders directly or from the two new remainders $N_{1}$ and $N_{2}$ of $N$ with two new moduli $q_{1}$ and $q_{2}$ with (39) and (41), respectively. For the robustness, as we mentioned earlier, the closed-form robust CRT and the results in Propositions 1 and 2 can not be applied to the four moduli and the four erroneous remainders directly since they do not satisfy the co-prime condition. In addition, according to our single stage robust CRT in Theorem 1 and Corollary 1 obtained in the previous section, its robustness bound would be $\tau<9 / 4$ (interestingly, for the 4 moduli, their ged is only 1). However, using the above grouping idea, the reconstruction of $N$ can be done in two stages: the first stage is to reconstruct $\hat{N}_{1}$ in (40) and $\hat{N}_{2}$ in (42) from the two groups, respectively; the second stage is to reconstruct $\hat{N}$ from its two possibly erroneous remainders $\hat{N}_{1}$ and $\hat{N}_{2}$ with two new moduli $q_{1}$ and $q_{2}$. From the second stage, using the known robust CRT again, we obtain

$$
\text { if } \tau<\operatorname{gcd}\left(q_{1}, q_{2}\right) / 4=18 / 4 \text {, then, }|N-\hat{N}| \leq \tau \text {. }
$$

Thus, we have a robust reconstruction too. In order to keep all inequalities (40), (42) and (43), one can see that with this twostage approach, the robustness bound on the remainder error level $\tau$ is $18 / 4$ which is surprisingly even better than $9 / 4$ that is the robustness bound in Corollary 1 using the single stage robust CRT for general moduli obtained in the previous section. This means that using two or more groups for a set of moduli may have a better robustness bound than that using a single group for the whole set of moduli. Clearly, for the better robustness, the way to group the moduli or remainders plays a very important role as one can see from the bounds above. Note that the robustness bound $\tau<9 / 4$ in Corollary 1 for the single stage robust CRT for the moduli set $\{180,220,486,513\}$ is only half of the robustness bound $\tau<18 / 4$ for the same moduli set $\{180,220,486,513\}$ but with the grouping and the two-stage approach. We next present our results for general cases. First, we consider the case of two groups and two stages.

Let $\left\{M_{1,1}, M_{1,2}, \ldots, M_{1, L_{1}}, M_{2,1}, M_{2,2}, \ldots, M_{2, L_{2}}\right\} \quad$ be the whole set of moduli that may not be necessarily all distinct. It is split to two groups with Group 1 of $L_{1}$ moduli: $0<M_{1,1}<M_{1,2}<\cdots<M_{1, L_{1}}$; and Group 2 of $L_{2}$ moduli: $0<M_{2,1}<M_{2,2}<\cdots<M_{2, L_{2}}$. These two groups do not have to be disjoint. Let $N$ be a positive integer, and $r_{1,1}, r_{1,2}, \ldots, r_{1, L_{1}}, r_{2,1}, r_{2,2}, \ldots, r_{2, L_{2}}$ be the corresponding remainders of $N$, i.e.,

$$
N=n_{j, i} M_{j, i}+r_{j, i}
$$

where $0 \leq r_{j, i}<M_{j, i}$ and $n_{j, i}$ is an unknown integer for $1 \leq i \leq L_{j}, j=1$ or 2 . As we know, $N$ can be uniquely reconstructed from its $L_{1}+L_{2}$ remainders if and only if $0 \leq$ $N<\operatorname{lcm}\left(\delta_{1}, \delta_{2}\right)$, where $\delta_{1} \triangleq \operatorname{lcm}\left(M_{1,1}, \ldots, M_{1, L_{1}}\right)$ and $\delta_{2} \triangleq \operatorname{lcm}\left(M_{2,1}, \ldots, M_{2, L_{2}}\right)$. The congruence system (44) can be converted into the following two-stage congruences.

For $j=1,2$, and Group $j$, we can write

$$
\left\{\begin{array}{l}
N_{j}=K_{j, i} M_{j, i}+r_{j, i} \\
0 \leq N_{j}<\delta_{j} \\
1 \leq i \leq L_{j} .
\end{array}\right.
$$

Then, the above $N_{1}$ and $N_{2}$ can be combined to form a new system of congruences:

$$
\left\{\begin{array}{l}
N=l_{1} \delta_{1}+N_{1} \\
N=l_{2} \delta_{2}+N_{2} \\
0 \leq N<\operatorname{lcm}\left(\delta_{1}, \delta_{2}\right)
\end{array}\right.
$$

When $\delta_{1}=\delta_{2}$, the two congruence equations are degenerated to a single equation and without loss of generality, we assume $\delta_{1} \neq$ $\delta_{2}$ here and such a similar degenerated case is not considered either in what follows in this paper. Replacing $N_{1}$ and $N_{2}$ in (46) by (45), we have

$$
\begin{cases}N=l_{1} \delta_{1}+K_{1, i} M_{1, i}+r_{1, i}, & 1 \leq i \leq L_{1} \\ N=l_{2} \delta_{2}+K_{2, i} M_{2, i}+r_{2, i}, & 1 \leq i \leq L_{2} \\ 0 \leq N<\operatorname{lcm}\left(\delta_{1}, \delta_{2}\right) . & \end{cases}
$$

It is not hard to see that

$$
\begin{aligned}
N & =l_{j} \delta_{j}+K_{j, i} M_{j, i}+r_{j, i} \\
& =\left(l_{j} \frac{\delta_{j}}{M_{j, i}}+K_{j, i}\right) M_{j, i}+r_{j, i},
\end{aligned}
$$


where

$$
K_{j, i}<\frac{\delta_{j}}{M_{j, i}}
$$

for $1 \leq i \leq L_{j}, j=1$ or 2 . Clearly,

$$
n_{j, i}=l_{j} \frac{\delta_{j}}{M_{j, i}}+K_{j, i}, \quad 1 \leq i \leq L_{j}, j=1,2 .
$$

From the known values of all the moduli $\left\{M_{j, i}\right\}$ and all the erroneous remainders $\left\{\tilde{r}_{j, i}\right\}$, if we can accurately determine $K_{j, i}$ and $l_{j}$, then we can accurately determine $n_{j, i}$. Thus, we propose the following algorithm to robustly reconstruct $N$, called two-stage robust CRT, when the remainders are erroneous.

- Step 1: Following the single stage robust CRT algorithm of Steps $1-5$ in Section II, calculate $\hat{K}_{j, i}$ for $1 \leq i \leq L_{j}$ in the system of congruence equations (45) from erroneous remainders $\left\{\tilde{r}_{i}\right\}$ for each $j=1,2$.

- Step 2: After obtaining $\hat{K}_{j, i}$ for $1 \leq i \leq L_{j}, j=1,2$, calculate the average estimate $\hat{N}_{j}$ of $\bar{N}_{j}$ for $j=1,2$ by (3) and (4):

$$
\hat{N}_{j}=\left[\frac{1}{L_{j}} \sum_{i=1}^{L_{j}}\left(\hat{K}_{j, i} M_{j, i}+\tilde{r}_{j, i}\right)\right],
$$

where $[\cdot]$ stands for the rounding integer (5).

- Step 3: Treating $\hat{N}_{1}$ and $\hat{N}_{2}$ as the new erroneous remainders in the system of congruence equations (46) and following the single stage robust CRT algorithm Steps 1-5 in Section II again, we calculate $\hat{l}_{1}$ and $\hat{l}_{2}$.

- Step 4: Calculate $\hat{n}_{j, i}$ for $1 \leq i \leq L_{j}$ and $j=1,2$ :

$$
\hat{n}_{j, i}=\hat{l}_{j} \frac{\delta_{j}}{M_{j, i}}+\hat{K}_{j, i}
$$

- Step 5: Calculate the average estimate $\hat{N}$ of the unknown integer $N$ :

$$
\hat{N}=\left[\frac{1}{L_{1}+L_{2}}\left(\sum_{j=1}^{2} \sum_{i=1}^{L_{j}}\left(\hat{n}_{j, i} M_{j, i}+\tilde{r}_{j, i}\right)\right)\right],
$$

where $[\cdot]$ stands for the rounding integer (5).

Then, we have the following result. For $j=1,2$, let $\tau_{j}$ denote the error level of the remainders $r_{j, i}$ in the $j$-th group, i.e.,

$$
\left|\Delta r_{j, i}\right|=\left|\tilde{r}_{j, i}-r_{j, i}\right| \leq \tau_{j}
$$

for $1 \leq i \leq L_{j}$ and

$$
G_{j}=\max _{1 \leq i \leq L_{j}} \min _{1 \leq q \neq i \leq L_{j}} \frac{\operatorname{gcd}\left(M_{j, i}, M_{j, q}\right)}{4} .
$$

Let

$$
G=\frac{\operatorname{gcd}\left(\delta_{1}, \delta_{2}\right)}{4}
$$

In the above, when the $j$-th group has only one modulus $M_{j, 1}$, then $G_{j}=\frac{M_{j, 1}}{4}$ and the corresponding $1 \mathrm{~cm}, \delta_{j}$, is just $M_{j, 1}$.

Theorem 2: If

$$
\begin{aligned}
& \left|\triangle r_{j, i}\right| \leq \tau_{j}<\min \left(G_{j}, G\right), \\
& \quad \text { for all } 1 \leq i \leq L_{j} \text { and } j=1,2,
\end{aligned}
$$

then, we can accurately determine the folding numbers $\hat{n}_{j, i}=$ $n_{j, i}$ for $1 \leq i \leq L_{j}, j=1,2$, and the average estimate $\hat{N}$ of the unknown integer $N$ in (50) satisfies

$$
|\hat{N}-N| \leq\left[\frac{L_{1} \tau_{1}+L_{2} \tau_{2}}{L_{1}+L_{2}}\right],
$$

where $[\cdot]$ stands for the rounding integer (5).

Proof: For $j=1,2$, according to Corollary 1, when $\left|\Delta r_{j, i}\right| \leq \tau_{j}<G_{j}$, we can accurately determine $K_{j, i}$ in the systems of congruence equations (45):

$$
K_{j, i}=\hat{K}_{j, i}, \quad \text { for } 1 \leq i \leq L_{j} .
$$

Furthermore, for the average estimates $\hat{N}_{1}$ and $\hat{N}_{2}$ in (48) in Step 2 above, we have

$$
\left|\Delta N_{j}\right|=\left|N_{j}-\hat{N}_{j}\right| \leq \tau_{j},
$$

which keeps the same error level as the remainders $r_{j, i}$ for $1 \leq$ $i \leq L_{j}, j=1,2$.

In the second stage (46), $\hat{N}_{1}$ and $\hat{N}_{2}$ become the erroneous remainders. To accurately determine $l_{1}$ and $l_{2}$, according to Proposition 2 or Corollary 1, the error levels should satisfy $\tau_{1}<$ $G=\frac{\operatorname{gcd}\left(\delta_{1}, \delta_{2}\right)}{4}$ and $\tau_{2}<G=\frac{\operatorname{gcd}\left(\delta_{1}, \delta_{2}\right)}{4}$, then

$$
l_{j}=\hat{l}_{j}, \quad \text { for } j=1,2 .
$$

Thus, combining with the first stage, we have the condition $\tau_{1}<$ $\min \left(G_{1}, G\right)$ and $\tau_{2}<\min \left(G_{2}, G\right)$ so that $K_{j, i}=\hat{K}_{j, i}$ and $l_{j}=\hat{l}_{j}$ for $1 \leq i \leq L_{j}, j=1,2$. Namely, we have $n_{j, i}=\hat{n}_{j, i}$ from (49).

After we accurately determine the folding numbers $n_{j, i}=$ $\hat{n}_{j, i}$ for $1 \leq i \leq L_{j}, j=1,2$, we can get the average estimate $\hat{N}$ in (50) of the unknown integer $N$, i.e.,

$$
\begin{aligned}
\hat{N} & =\left[\frac{1}{L_{1}+L_{2}}\left(\sum_{j=1}^{2} \sum_{i=1}^{L_{j}}\left(\hat{n}_{j, i} M_{j, i}+\tilde{r}_{j, i}\right)\right)\right] \\
& =N+\left[\frac{1}{L_{1}+L_{2}}\left(\sum_{i=1}^{L_{1}}\left(\Delta r_{1, i}\right)+\sum_{i=1}^{L_{2}}\left(\Delta r_{2, i}\right)\right)\right] .
\end{aligned}
$$

From $\left|\Delta r_{j, i}\right| \leq \tau_{j}$ for $1 \leq i \leq L_{j}, j=1,2$, we can easily obtain

$$
|\hat{N}-N| \leq\left[\frac{L_{1} \tau_{1}+L_{2} \tau_{2}}{L_{1}+L_{2}}\right] .
$$

Therefore the proof is completed.

The above results for two groups of moduli can be easily generalized to a general number of groups of moduli by using Corollary 1 twice for the two stages of the congruence equations as follows.

Assume that there are $s$ groups of moduli with $s>2$. For every $1 \leq j \leq s$, the elements in the $j$-th group are denoted as $0<M_{j, 1}<M_{j, 2}<\cdots<M_{j, L_{j}}$, let $\delta_{j} \triangleq \operatorname{lcm}\left(M_{j, 1}, M_{j, 2}, \ldots, M_{j, L_{j}}\right)$ and $\tau_{j}$ denote the error level of the remainders $r_{j, i}, 1 \leq i \leq L_{j}$, in the $j$-th group, and when the $j$-th group has more than one element, define

$$
G_{j}=\max _{1 \leq i \leq L_{j}} \min _{1 \leq q \neq i \leq L_{j}} \frac{\operatorname{gcd}\left(M_{j, i}, M_{j, q}\right)}{4} .
$$


If the $j$-th group has only one element, $M_{j, 1}$, define $G_{j}=\frac{M_{j, 1}}{4}$. Let

$$
G=\max _{1 \leq i \leq s} \min _{1 \leq q \neq i \leq s} \frac{\operatorname{gcd}\left(\delta_{i}, \delta_{q}\right)}{4} .
$$

Then, we have the following result.

Theorem 3: If

$$
\begin{aligned}
\left|\triangle r_{j, i}\right| & \leq \tau_{j}<\min \left(G_{j}, G\right), \\
& \text { for all } 1 \leq i \leq L_{j}, 1 \leq j \leq s,
\end{aligned}
$$

then, we can accurately determine the folding numbers $\hat{n}_{j, i}=$ $n_{j, i}$ for $1 \leq i \leq L_{j}$ and $1 \leq j \leq s$, and thus we can robustly reconstruct $\hat{N}$ as an estimate of $N$ when $0 \leq N<$ $\operatorname{lcm}\left(\delta_{1}, \delta_{2}, \ldots, \delta_{s}\right)$ :

$$
\begin{aligned}
\hat{N} & =\left[\frac{1}{\sum_{j=1}^{s} L_{j}} \sum_{j=1}^{s} \sum_{i=1}^{L_{j}}\left(\hat{n}_{j, i} M_{j, i}+\tilde{r}_{j, i}\right)\right], \\
|\hat{N}-N| & \leq\left[\frac{\sum_{j=1}^{s} L_{j} \tau_{j}}{\sum_{j=1}^{s} L_{j}}\right] .
\end{aligned}
$$

Proof: The proof is similar to the proof of Theorem 2. In the first stage, via (59) we can accurately determine the folding numbers $K_{j, i}$ for $1 \leq i \leq L_{j}, 1 \leq j \leq s$, and obtain the robust estimates $\hat{N}_{j}$ of $N_{j}$ for the $j$-th group with the error bound $\mid \hat{N}_{j}-$ $N_{j} \mid \leq \tau_{j}<\min \left(G_{j}, G\right)$, where $0 \leq N_{j}<\delta_{j}$ for all $1 \leq j \leq$ $s$.

Then, in the second stage we take these estimates $\hat{N}_{j}$ as erroneous remainders and $\delta_{j}$ as moduli for $1 \leq j \leq s$ to form a new congruence system. Applying the result of Corollary 1 again, we can accurately determine the unknown folding numbers $l_{j}$ for $1 \leq j \leq s$. By that, we can accurately determine $n_{j, i}$ for $1 \leq i \leq L_{j}$ with $1 \leq j \leq s$.

Lastly, once we get the accurate values of $n_{j, i}$, the average estimate $\hat{N}$ of $N$ can be found. The error bound of $N$ is proved similar to Theorem 2. Hence, the theorem is proved.

Similar to Corollary 3 for the single stage robust CRT, in the second stage with moduli $\delta_{j}$ and erroneous remainders $\hat{N}_{j}$ for $1 \leq j \leq s$, we can also individually consider the remainder error level for each remainder $\hat{N}_{j}$ with respect to modulus $\delta_{j}$ and have the following result.

Corollary 5: Assume that the reference modulus is $\delta_{k}$ for some $k$ with $1 \leq k \leq s$, which satisfies

$$
G=\min _{1 \leq q \neq k \leq s} \frac{\operatorname{gcd}\left(\delta_{k}, \delta_{q}\right)}{4}=\max _{1 \leq i \leq s} \min _{1 \leq q \neq i \leq s} \frac{\operatorname{gcd}\left(\delta_{i}, \delta_{q}\right)}{4}
$$

and its corresponding remainder error bound $\tau_{k}<G$. If

$$
\begin{aligned}
& \left|\triangle r_{k, i}\right| \leq \tau_{k}<\min \left(G_{k}, G\right), \quad \text { for all } 1 \leq i \leq L_{k}, \quad \text { (63) } \\
& \left|\triangle r_{j, i}\right| \leq \tau_{j}<\min \left(G_{j}, \frac{\operatorname{gcd}\left(\delta_{j}, \delta_{k}\right)}{2}-\min \left(G_{k}, G\right)\right),
\end{aligned}
$$

for all $1 \leq i \leq L_{j}$ and $1 \leq j \neq k \leq s$, then we can accurately determine the folding numbers $\hat{n}_{j, i}=n_{j, i}$ for $1 \leq i \leq L_{j}$ and $1 \leq j \leq s$.

Example 3: Given three groups of moduli: $\{21 \cdot 5,21 \cdot 7\},\{27$. $7,27 \cdot 13\}$, and $\{28 \cdot 15,28 \cdot 11\}$. We can get $G_{1}=21 / 4, G_{2}=$
$27 / 4, G_{3}=28 / 4$ and $G=21 / 4$. So, from Theorem 3, we obtain the robustness bounds: $\tau_{1}<\min \left(G_{1}, G\right)=21 / 4, \tau_{2}<$ $\min \left(G_{2}, G\right)=21 / 4$ and $\tau_{3}<\min \left(G_{3}, G\right)=21 / 4$. If we use the result of Corollary 5 , we can get a better error bound for some groups as follows: $\tau_{1} \leq 21 / 4, \tau_{2} \leq 21 / 4$ and $\tau_{3}<28 / 4$.

For a given set of $L$ moduli $M_{i}, 1 \leq i \leq L$, there are many different grouping methods of the remainders, and therefore, many ways to robustly reconstruct the unknown integer from its erroneous remainders. Let us see an example.

Example 4: Consider the moduli set $\left\{M_{1}, M_{2}, M_{3}, M_{4}\right\}=$ $\{48 \cdot 4,48 \cdot 3,56 \cdot 3,56 \cdot 2\}$. First, consider all the moduli as a single group and we obtain the robustness bound 24/4 according to Corollary 1 . Second, we group the four moduli into two groups $\{48 \cdot 4,48 \cdot 3\}$ and $\{56 \cdot 3,56 \cdot 2\}$ with $G_{1}=$ $48 / 4, G_{2}=56 / 4$ and $G=\frac{\operatorname{gcd}\left(\delta_{1}, \delta_{2}\right)}{4}=48 / 4$ in Theorem 2 . Accordingly, the robustness bound in this case is $\tau_{1}<48 / 4$ and $\tau_{2}<48 / 4$. Lastly, if we group the four moduli into another two groups $\{48 \cdot 4,56 \cdot 2\}$ and $\{48 \cdot 3,56 \cdot 3\}$ with $G_{1}=16 / 4, G_{2}=$ $24 / 4$ and $G=336 / 4$, then, the robustness bound in this case is $\tau_{1}<16 / 4$ and $\tau_{2}<24 / 4$.

From this example, we can see that different grouping methods lead to different robustness bounds. Compared with the robustness bound by using a single stage robust CRT for the whole set of moduli, sometimes a grouping can enlarge the robustness bound while sometimes a grouping may decrease the robustness bound. Thus, another question is whether there exists a proper grouping method to ensure the robustness bound larger than that in Corollary 1 using the single stage robust CRT. We next present a result that tells us when there exists a grouping method with a better robustness bound for remainders in some groups using a two-stage robust CRT than that using the single stage robust CRT.

Corollary 6: For a given set of $L$ moduli $\left\{M_{i}, i=1, \ldots, L\right\}$, the robustness bound can not be enlarged for remainders in any group by using a two-stage robust CRT with a grouping method of the moduli if and only if it is the case of [13], i.e., the remaining factors $\Gamma_{i}$ of the moduli $M_{i}=M \Gamma_{i}$ divided by their $\operatorname{gcd} M, 1 \leq i \leq L$, are co-prime.

Proof: It is easy to prove the sufficiency as follows. When the moduli $M_{i}$ satisfy the constraint in [13], i.e., $\Gamma_{i}$ are co-prime, its robustness bound using the single stage robust CRT with a single group moduli is $M / 4$. On the other hand, from Theorem 3, each $G_{j}$ of any grouping and $G$ are both $M / 4$. Hence, we cannot enlarge the robustness bound in this case.

We next prove the necessity. Assume that the robustness bound for remainders in any group can not be enlarged by the two-stage robust CRT with a grouping method of the moduli over the robustness bound of the single stage robust CRT of the whole set of the moduli. Denote $\operatorname{gcd}\left(M_{i}, M_{q}\right)=m_{i q}$. Without loss of generality, we can assume

$$
\begin{gathered}
\frac{m_{1 L}}{4}=\frac{\operatorname{gcd}\left(M_{1}, M_{L}\right)}{4}=\max _{\substack{1 \leq i \leq L \\
\text { and }}} \min _{1 \leq q \neq i \leq L} \frac{\operatorname{gcd}\left(M_{i}, M_{q}\right)}{4}, \\
m_{12} \geq m_{13} \geq \cdots \geq m_{1 L} .
\end{gathered}
$$

Thus, according to Corollary 1, its robustness bound using the single stage robust CRT with a single group moduli is $m_{1 L} / 4$. We then have the following two cases. 
Case I: There exists one $q$ with $2 \leq q<L$ such that

$$
m_{12} \geq \cdots \geq m_{1 q}>m_{1(q+1)} \geq \cdots \geq m_{1 L} .
$$

If we group the moduli as Group 1: $\left\{M_{1}, \ldots, M_{q}\right\}$; and Group 2: $\left\{M_{1}, M_{q+1}, \ldots, M_{L}\right\}$. With this grouping, we have that $G_{1}>m_{1 L} / 4$ and $G_{2} \geq m_{1 L} / 4, G \geq M_{1} / 4>m_{1 L} / 4$. Thus, we obtain $\tau_{1}<\min \left\{G_{1}, G\right\}, \tau_{2}<\min \left\{G_{2}, G\right\}$, where $\min \left\{G_{1}, G\right\}>m_{1 L} / 4=\tau$, which contradicts with the assumption that we cannot enlarge the robustness bound for the remainders in Group 1 using a two-stage robust CRT. This proves that $m_{12}=m_{13}=\cdots=m_{1 L}$.

Case II: Under the condition of $m_{12}=m_{13}=\cdots=m_{1 L}=$ $M$, we know that any $m_{i q}=\operatorname{gcd}\left(M_{i}, M_{q}\right) \geq M$, since $M$ is a factor of all the moduli $M_{i}$. Suppose that there exists one $m_{i q}>M$ with $q \neq i \neq 1$. We can group the moduli as Group 1: $\left\{M_{i}, M_{q}\right\}$, and Group 2: $\left\{M_{i},\left\{M_{i_{1}}\right\}_{i_{1} \neq q \text { or } i}\right\}$. Similar to Case I, $G_{1}>M / 4, G_{2} \geq M / 4$ and $G \geq M_{i} / 4>M / 4$. So, we can enlarge the robustness bound for the remainders in Group 1 by using the two-stage robust CRT with this grouping. This also contradicts with the assumption.

From the above two cases we conclude that $m_{i q}=M$ for all $1 \leq i \neq q \leq L$, i.e., it is the case of [13].

Now, we give an explicit example. Suppose there are a set of moduli with the form of $\left\{M_{1} K_{1}, M_{2} K_{1}, M_{2} K_{2}, M_{2} K_{3}\right\}$, where $M_{1}, K_{1}, K_{2}, K_{3}$ are co-prime. According to Corollary 1, the robustness bound using the single stage robust CRT is $\tau<$ $\min \left(M_{2}, K_{1} \operatorname{gcd}\left(M_{1}, M_{2}\right)\right) / 4$. If the moduli are grouped into two groups as $\left\{M_{1} K_{1}, M_{2} K_{1}\right\}$ and $\left\{M_{2} K_{1}, M_{2} K_{2}, M_{2} K_{3}\right\}$. Then, according to Theorem 2, its robustness bound is $\tau_{1}<$ $K_{1} \operatorname{gcd}\left(M_{1}, M_{2}\right) / 4$ and $\tau_{2}<M_{2} / 4$, one of which is greater than the robustness bound $\min \left(M_{2}, K_{1} \operatorname{gcd}\left(M_{1}, M_{2}\right)\right) / 4$ when $M_{2} \neq K_{1} \operatorname{gcd}\left(M_{1}, M_{2}\right)$.

Example 5: Let $M_{1}=8, M_{2}=14, K_{1}=3, K_{2}=5$, and $K_{3}=7$. Then we can calculate $\tau_{1}<6 / 4$ and $\tau_{2}<14 / 4$ from the two-stage robust CRT. One can see that $\tau_{2}<14 / 4$ is significantly greater than $\tau<6 / 4$ using the single stage robust CRT.

From Corollary 6 , one can see that as long as $\Gamma_{i}$ in moduli $M_{i}$ are not all co-prime, using a two-stage robust CRT with some grouping method has a larger robustness bound for remainders in some groups than the single stage robust CRT does. In the same way, we may treat $\left\{\delta_{1}, \delta_{2}, \ldots, \delta_{s}\right\}$ as a new set of moduli and group it again so that the single stage robust CRT is applied three times with the following result. We call it three-stage robust CRT.

Let us split $\left\{\delta_{1}, \delta_{2}, \ldots, \delta_{s}\right\}$ in Theorem 3 to $k$ groups. For every $1 \leq t \leq k$, the elements in the $t$-th group are denoted as $0<\delta_{t, 1}<\cdots<\delta_{t, y_{t}}$, let $\xi_{t} \triangleq \operatorname{lcm}\left(\delta_{t, 1}, \ldots, \delta_{t, y_{t}}\right)$ and define

$$
\Upsilon_{t}=\max _{1 \leq i \leq y_{t}} \min _{1 \leq q \neq i \leq y_{t}} \frac{\operatorname{gcd}\left(\delta_{t, i}, \delta_{t, q}\right)}{4} .
$$

Let

$$
\Upsilon=\max _{1 \leq i \leq k} \min _{1 \leq q \neq i \leq k} \frac{\operatorname{gcd}\left(\xi_{i}, \xi_{q}\right)}{4} .
$$

We then have the following result.
Theorem 4: If for all $1 \leq i \leq L_{j}, 1 \leq j \leq s$ and $1 \leq t \leq k$,

$$
\begin{aligned}
\left|\triangle r_{j, i}\right| & \leq \tau_{j} \\
& <\min \left(G_{j}, \min _{t}\left\{\Upsilon_{t}: \delta_{j} \in\left\{\delta_{t, 1}, \ldots, \delta_{t, y_{t}}\right\}\right\}, \Upsilon\right),
\end{aligned}
$$

then, we can accurately determine the folding numbers $\hat{n}_{j, i}=n_{j, i}$ for $1 \leq i \leq L_{j}, 1 \leq j \leq s$, and thus we can robustly reconstruct $\hat{N}$ as an estimate of $N$ when $0 \leq N<\operatorname{lcm}\left(\delta_{1}, \delta_{2}, \ldots, \delta_{s}\right)$ :

$$
\begin{gathered}
\hat{N}=\left[\frac{1}{\sum_{j=1}^{s} L_{j}} \sum_{j=1}^{s} \sum_{i=1}^{L_{j}}\left(\hat{n}_{j, i} M_{j, i}+\tilde{r}_{j, i}\right)\right], \\
|\hat{N}-N| \leq\left[\frac{\sum_{j=1}^{s} L_{j} \tau_{j}}{\sum_{j=1}^{s} L_{j}}\right] .
\end{gathered}
$$

Proof: The congruence system

$$
N=n_{j, i} M_{j, i}+r_{j, i},
$$

where $0 \leq r_{j, i}<M_{j, i}$ for $1 \leq i \leq L_{j}, 1 \leq j \leq s$ and $0 \leq$ $N<\operatorname{lcm}\left(\delta_{1}, \delta_{2}, \ldots, \delta_{s}\right)$, can be converted into the following three-stage congruences.

For $1 \leq j \leq s$, and Group $j$ in the first stage, we can write

$$
\left\{\begin{array}{l}
N_{j}=K_{j, i} M_{j, i}+r_{j, i} \\
0 \leq N_{j}<\delta_{j} \\
1 \leq i \leq L_{j} .
\end{array}\right.
$$

In the second stage,

$$
\left\{\begin{array}{l}
P_{t}=H_{t, i} \delta_{t, i}+N_{t, i} \\
0 \leq P_{t}<\xi_{t} \\
1 \leq i \leq y_{t} \\
1 \leq t \leq k
\end{array}\right.
$$

Then, in the third stage, we can write

$$
\left\{\begin{array}{l}
N=l_{t} \xi_{t}+P_{t} \\
0 \leq N<\operatorname{lcm}\left(\xi_{1}, \ldots, \xi_{k}\right) \\
1 \leq t \leq k .
\end{array}\right.
$$

As long as we can accurately determine all of $K_{j, i}, H_{t, i}$ and $l_{t}$ in each congruence system, we can then accurately determine $n_{j, i}$. According to conditions (67), we can accurately determine $K_{j, i}$ for $1 \leq i \leq L_{j}, 1 \leq j \leq s$ and get the error bound

$$
\begin{aligned}
\left|\hat{N}_{j}-N_{j}\right| & \leq \tau_{j} \\
& <\min \left(G_{j}, \min _{t}\left\{\Upsilon_{t}: \delta_{j} \in\left\{\delta_{t, 1}, \ldots, \delta_{t, y_{t}}\right\}\right\}, \Upsilon\right) .
\end{aligned}
$$

Next, in each group of the second stage we take these estimates $\hat{N}_{j}$ as erroneous remainders and $\delta_{j}$ as moduli. Applying the result of Corollary 1 , we can accurately determine $H_{t, i}$, and also get the robust estimate $\hat{P}_{t}$ satisfying

$$
\begin{aligned}
\mid \hat{P}_{t}- & P_{t} \mid \\
& <\min \left(G_{j}, \min _{t}\left\{\Upsilon_{t}: \delta_{j} \in\left\{\delta_{t, 1}, \ldots, \delta_{t, y_{t}}\right\}\right\}, \Upsilon\right) .
\end{aligned}
$$


Similarly, treat the estimates $\hat{P}_{t}$ as the erroneous remainders and $\xi_{t}$ as moduli in the third stage. Since $\left|\hat{P}_{t}-P_{t}\right|<\Upsilon$, from Corollary 1 again, we can accurately determine $l_{t}$. Once we accurately determine these unknown folding numbers in each congruence system, we can accurately determine $n_{j, i}$ and then obtain the robust estimate $\hat{N}$ of the unknown integer $N$. As for the error bound of the estimate $\hat{N}$, the proof is the same to that of Theorem 2. Therefore, we complete the proof.

Example 6: Consider a given set of moduli $\{96 \cdot 2,96 \cdot 3,72$. $3,72 \cdot 5,64 \cdot 5,64 \cdot 7\}$. Treating them as one group and using the single stage robust CRT, we get its error bound $\tau$ for the remainders satisfying $\tau<32 / 4$. If we split the moduli to three groups: $\{96 \cdot 2,96 \cdot 3\},\{72 \cdot 3,72 \cdot 5\}$ and $\{64 \cdot 5,64 \cdot 7\}$, we get $G_{1}=96 / 4, G_{2}=72 / 4, G_{3}=64 / 4, \delta_{1}=96 \cdot 2 \cdot 3, \delta_{2}=72$. $3 \cdot 5, \delta_{3}=64 \cdot 5 \cdot 7$ and $G=64 / 4$. By using the two-stage robust CRT, we can get the error bounds $\tau_{j}$ for the remainders in Group $j$ for $j=1,2,3$ satisfying $\tau_{1}<\min \left(G_{1}, G\right)=64 / 4, \tau_{2}<$ $\min \left(G_{2}, G\right)=64 / 4$ and $\tau_{3}<\min \left(G_{3}, G\right)=64 / 4$, all of which are larger than the bound 32/4 in the single stage robust CRT. If we use the three-stage robust CRT and split $\left\{\delta_{1}, \delta_{2}, \delta_{3}\right\}$ to two groups again: $\left\{\delta_{1}, \delta_{2}\right\},\left\{\delta_{3}\right\}$, we can get $\Upsilon_{1}=72 / 4, \Upsilon=$ $320 / 4$. So, in this three-stage robust CRT, the error bounds satisfy $\tau_{1}<\min \left(G_{1}, \Upsilon_{1}, \Upsilon\right)=72 / 4, \tau_{2}<\min \left(G_{2}, \Upsilon_{1}, \Upsilon\right)=72 / 4$ and $\tau_{3}<\min \left(G_{3}, \Upsilon\right)=64 / 4$. Compared with the two-stage robust CRT, we increase the robustness bounds in Group 1 and Group 2 from 64/4 to 72/4 by using the three-stage robust CRT.

The above three-stage robust CRT can be easily generalized to a multi-stage robust CRT with more than three stages. Although we can use a multi-stage robust CRT with some grouping methods to obtain a larger robustness bound for remainders in some groups, there are some challenges about how to choose moduli in a group and how many groups and stages we should split in order to find a better robustness bound such that we can enlarge all the robustness bounds in every group.

Let us first look at the simplest case when there are only three moduli $\left\{M_{1}, M_{2}, M_{3}\right\}$. Without loss of generality, we can assume that $\operatorname{gcd}\left(M_{1}, M_{2}\right) \geq \operatorname{gcd}\left(M_{1}, M_{3}\right) \geq \operatorname{gcd}\left(M_{2}, M_{3}\right)$. Regarding the three moduli as one group and by Corollary 1 , the robustness bound is $\operatorname{gcd}\left(M_{1}, M_{3}\right) / 4$. Since $\operatorname{gcd}\left(M_{3}, M_{2}\right) \leq$ $\operatorname{gcd}\left(M_{3}, M_{1}\right)$, if we want to obtain a robustness bound strictly larger than $\operatorname{gcd}\left(M_{1}, M_{3}\right) / 4$, the modulus $M_{3}$ must independently form an individual group by itself, and in the meantime it does not allow other groups to include $M_{3}$. Thus, there is only one possible grouping method as $\left\{M_{3}\right\}$ and $\left\{M_{1}, M_{2}\right\}$. The robustness bound therein is $\tau_{1}<\operatorname{gcd}\left(M_{3}, \operatorname{lcm}\left(M_{1}, M_{2}\right)\right) / 4$ and $\tau_{2}<\min \left(\operatorname{gcd}\left(M_{1}, M_{2}\right), \operatorname{gcd}\left(M_{3}, \operatorname{lcm}\left(M_{1}, M_{2}\right)\right)\right) / 4$, which may be both larger than $\operatorname{gcd}\left(M_{1}, M_{3}\right) / 4$. Otherwise, we have to group them as $\left\{M_{1}, M_{3}\right\}$ and $\left\{M_{1}, M_{2}\right\}$ and in this way we may only enlarge one group's (not every group's) robustness bound as what is used in the proof of Corollary 6 .

Example 7: When $M_{1}=560, M_{2}=480, M_{3}=210$, we can see that $\operatorname{gcd}\left(M_{1}, M_{2}\right)=\operatorname{gcd}(560,480)=80$, $\operatorname{gcd}\left(M_{1}, M_{3}\right)=\operatorname{gcd}(560,210)=70$ and $\operatorname{gcd}\left(M_{2}, M_{3}\right)=$ $\operatorname{gcd}(480,210)=30$. Regarding these three moduli as a single group, the robustness bound is $\operatorname{gcd}\left(M_{1}, M_{3}\right) / 4=$ $\operatorname{gcd}(560,210) / 4=70 / 4$. In order to find a larger robustness bound, we just only consider the robustness bound of the case of two groups: $\left\{M_{3}\right\}$ and $\left\{M_{1}, M_{2}\right\}$. We can get $\tau_{1}<210 / 4$ and $\tau_{2}<80 / 4$, which are all larger than 70/4.
The above special case is about only three moduli's grouping. When the number of given moduli is larger, it becomes more complicated. In the next section, we analyze some special cases.

\section{An Algorithm For Grouping Moduli in Two-Stage ROBUST CRT}

From the above study, one may see that for a given set of moduli, although its determinable range for an integer from its remainders is fixed, i.e., the $1 \mathrm{~cm}$ of all the moduli, the robustness bounds for an erroneous remainder and a reconstructed integer depend on a reconstruction algorithm from erroneous remainders, which depends on the grouping of the moduli in a multi-stage robust CRT. For a general set of moduli, it is not obvious on how to group them in a multi-stage (or even two-stage) robust CRT, in particular when the number of moduli is not small. In this section, based on Theorem 3 for the two-stage robust CRT, we propose an algorithm for grouping a general set of moduli to possibly obtain a larger robustness bound for remainders in every group than that in Corollary 1 for the single stage robust CRT.

For a given set of moduli $\mathcal{M}=\left\{M_{1}, M_{2}, \ldots, M_{L}\right\}, L \geq 3$, we first assume that the set of moduli does not include any pair of $M_{i_{1}}$ and $M_{i_{2}}$ satisfying $M_{i_{1}}=n M_{i_{2}}$, because Corollary 2 has told us that such a redundant modulus $M_{i_{2}}$ does not help to increase the determinable range of $N, 0 \leq N<$ $\operatorname{lcm}\left(M_{1}, M_{2}, \ldots, M_{L}\right)$ nor the robustness bound in a single stage robust CRT. From condition (59) we need to assure that all $G_{j}$ in (57) and $G$ in (58) after a grouping strictly greater than $\Theta \triangleq \max _{1<i<L} \min _{1<j \neq i<L} \frac{\operatorname{gcd}\left(M_{i}, M_{j}\right)}{4}$ in Corollary 1 for the single stage robust CRT. Then, we have an algorithm as follows.

1) For each $M_{i}, 1 \leq i \leq L$, find all $M_{j}, 1 \leq j \neq i \leq$ $L$, satisfying $\frac{\operatorname{gcd}\left(M_{j}, \overline{M_{i}}\right)}{4}>\Theta$. Then, with $M_{i}$, form the corresponding set $\mathcal{M}_{i}$ :

$$
\mathcal{M}_{i}=\left\{M_{i}, M_{j}: \frac{\operatorname{gcd}\left(M_{j}, M_{i}\right)}{4}>\Theta\right\} .
$$

Thus, with each set $\mathcal{M}_{i}$, we have

$$
G_{i} \geq \min _{M_{j} \in \mathcal{M}_{i}} \frac{\operatorname{gcd}\left(M_{j}, M_{i}\right)}{4}>\Theta
$$

If modulus $M_{i}$ satisfies $\frac{\operatorname{gcd}\left(M_{j}, M_{i}\right)}{4} \leq \Theta$ for all $M_{j}, 1 \leq$ $j \neq i \leq L$, then we let $\mathcal{M}_{i}=\left\{M_{i}\right\}$.

2) Among all of the $L$ sets $\mathcal{M}_{i}$ for $1 \leq i \leq L$, there may be one or more pairs, $\mathcal{M}_{i_{1}}$ and $\mathcal{M}_{i_{2}}$, satisfying $\mathcal{M}_{i_{1}} \subseteq \mathcal{M}_{i_{2}}$. In this case, we can delete the smaller set $\mathcal{M}_{i_{1}}$ and only keep the larger set $\mathcal{M}_{i_{2}}$.

3) After Step 2), from the remaining sets of $\left\{\mathcal{M}_{i}\right\}$, we find all such combinations of $\left\{\mathcal{M}_{i_{1}}, \mathcal{M}_{i_{2}}, \ldots, \mathcal{M}_{i_{l}}\right\}$ that $\bigcup_{j=1}^{l} \mathcal{M}_{i_{j}}$ exactly includes all moduli $\mathcal{M}$. In other words, if anyone $\mathcal{M}_{i_{s}}$ for $1 \leq s \leq l$ is deleted from a combination $\left\{\mathcal{M}_{i_{1}}, \mathcal{M}_{i_{2}}, \ldots, \mathcal{M}_{i_{l}}\right\}$, then $\bigcup_{j=1 \& j \neq s}^{l} \mathcal{M}_{i_{j}}$ is a proper subset of $\mathcal{M}$, i.e.,

$$
\mathcal{M} \neq \bigcup_{j=1 \& j \neq s}^{l} \mathcal{M}_{i_{j}} \subset \mathcal{M} \subseteq \bigcup_{j=1}^{l} \mathcal{M}_{i_{j}} .
$$


4) As for every combination in the above, treat each $\mathcal{M}_{i_{j}}$ as a small group and calculate its $1 \mathrm{~cm}$ as $\delta_{i_{j}}, 1 \leq j \leq l$. Then, check whether

$$
G=\max _{1 \leq p \leq l} \min _{1 \leq q \neq p \leq l} \frac{\operatorname{gcd}\left(\delta_{i_{p}}, \delta_{i_{q}}\right)}{4}>\Theta
$$

If there is one combination $\left\{\mathcal{M}_{i_{j}}\right\}$ as above to make inequality (78) hold, then every $\min \left(G_{i_{j}}, G\right), 1 \leq j \leq l$, is strictly greater than $\Theta$. According to (59) in Theorem 3, one can see that this combination is just a grouping as desired and it enlarges a robustness bound for remainders in every group by using the two-stage robust CRT. Otherwise, if for every possible combination in Step 3), inequality (78) does not hold, then it is said that we fail to use this algorithm to enlarge a robustness bound for remainders in every group by using the two-stage robust CRT.

Let us first consider the above grouping algorithm for the case of [13], i.e., the remaining factors $\Gamma_{i}$ of the moduli $M_{i}=M \Gamma_{i}$ divided by their gcd $M, 1 \leq i \leq L$, are co-prime. First, we find all $\mathcal{M}_{i}=\left\{M_{i}\right\}, 1 \leq i \leq L$. Next, there is only one combination $\left\{\mathcal{M}_{1}, \mathcal{M}_{2}, \ldots, \mathcal{M}_{L}\right\}$ satisfying (77), and we treat each $\mathcal{M}_{i}=\left\{M_{i}\right\}$ as one group, then calculate $G=M / 4$ in (78), which equals to $\Theta=M / 4$. In conclusion, we fail to find a grouping to enlarge a robustness bound for remainders in every group by using the two-stage robust CRT, which can be also confirmed from the earlier result in Corollary 6 . Next, we give a positive example.

Example 8: Consider a set of moduli $\{210 M, 143 M, 77 M, 128 M, 81 M, 125 M, 169 M\}$, where $M$ is an integer. As one group, using the single stage robust CRT, its robustness bound is $\Theta=M / 4$. According to the above grouping algorithm, find 7 sets: $\mathcal{M}_{1}=\{210 M, 77 M, 128 M, 81 M, 125 M\}$, $\mathcal{M}_{2}=\{143 M, 77 M, 169 M\}, \mathcal{M}_{3}=\{77 M, 210 M, 143 M\}$, $\mathcal{M}_{4}=\{128 M, 210 M\}, \mathcal{M}_{5}=\{81 M, 210 M\}, \mathcal{M}_{6}=$ $\{125 M, 210 M\}, \mathcal{M}_{7}=\{169 M, 143 M\}$. Among them, there are only four combinations satisfying (77) as follows: $\left\{\mathcal{M}_{1}, \mathcal{M}_{2}\right\},\left\{\mathcal{M}_{1}, \mathcal{M}_{7}\right\},\left\{\mathcal{M}_{2}, \mathcal{M}_{4}, \mathcal{M}_{5}, \mathcal{M}_{6}\right\}$, $\left\{\mathcal{M}_{3}, \mathcal{M}_{4}, \mathcal{M}_{5}, \mathcal{M}_{6}, \mathcal{M}_{7}\right\}$. Then, check whether one of the above four combinations satisfies inequality (78). Fortunately, for the first combination $\left\{\mathcal{M}_{1}, \mathcal{M}_{2}\right\}$, inequality (78) holds. We can calculate $G_{1}=2 M / 4, G_{2}=11 M / 4, G=7 M / 4$, all of which are strictly greater than $M / 4$. Thus, we have obtained a grouping method of the moduli to enlarge a robustness bound for remainders in every group by using the two-stage robust CRT.

Remark 2: As one can see in the proof of Corollary 6 and in the above algorithm and examples, a modulus $M_{i}$ may be repeatedly used in more than one groups in the two-stage robust CRT. Its aim is to make $G$ and $G_{j}$ after grouping greater than or equal to the robustness bound by using the single stage robust CRT for the whole set of moduli. Recall the case of grouping a set of three moduli $\left\{M_{1}, M_{2}, M_{3}\right\}$. Assume $\operatorname{gcd}\left(M_{1}, M_{2}\right)>\operatorname{gcd}\left(M_{1}, M_{3}\right)>\operatorname{gcd}\left(M_{2}, M_{3}\right)$. From Corollary 1, the robustness bound for using the single stage robust CRT is $\frac{\operatorname{gcd}\left(M_{1}, M_{3}\right)}{4}$. According to the above grouping moduli algorithm in the two-stage robust CRT, they are split to two groups: $\left\{M_{1}, M_{2}\right\}$ and $\left\{M_{3}\right\}$. One can see that
$G_{1}=\frac{\operatorname{gcd}\left(M_{1}, M_{2}\right)}{4}, G_{2}=\frac{M_{3}}{4}, \delta_{1}=\operatorname{lcm}\left(M_{1}, M_{2}\right), \delta_{2}=M_{3}$ and $G=\frac{\operatorname{gcd}\left(\delta_{1}, \delta_{2}\right)}{4}$. In this grouping method, the robustness bound for remainders in group $\left\{M_{1}, M_{2}\right\}$ is $\min \left(G_{1}, G\right)$ and the robustness bound for remainders in group $\left\{M_{3}\right\}$ is $\min \left(G_{2}, G\right)$. As $G_{1}$ and $G_{2}$ are greater than $\frac{\operatorname{gcd}\left(M_{1}, M_{3}\right)}{4}$, a robustness bound for remainders in each group depends on the value of $G$. When $G=\frac{\operatorname{gcd}\left(\delta_{1}, \delta_{2}\right)}{4}$ is less than $\frac{\operatorname{gcd}\left(M_{1}, M_{3}\right)}{4}$, a robustness bound for remainders in each group is worse than that for the single stage robust CRT. Thus, we should repeat modulus $M_{1}$ in group $\left\{M_{3}\right\}$, and the two groups become $\left\{M_{1}, M_{2}\right\}$ and $\left\{M_{1}, M_{3}\right\}$. In this way, we enlarge a robustness bound for group $\left\{M_{1}, M_{2}\right\}$ and keep the robustness bound for group $\left\{M_{1}, M_{3}\right\}$ non-changed. On the other hand, when $G=\frac{\operatorname{gcd}\left(\delta_{1}, \delta_{2}\right)}{4}$ is larger than $\frac{\operatorname{gcd}\left(M_{1}, M_{3}\right)}{4}$, we do not need to repeat modulus $M_{1}$, since the robustness bound for group $\left\{M_{1}, M_{2}\right\}$ and the robustness bound for group $\left\{M_{3}\right\}$ are both greater than $\frac{\operatorname{gcd}\left(M_{1}, M_{3}\right)}{4}$. This example tells us that, to enlarge the robustness bound, whether a modulus $M_{i}$ is repeatedly used or not in multiple groups depends on the grouping method and the set of moduli. Repeating a modulus, sometimes, may help to enlarge the robustness bound but sometimes may not.

\section{SimULATIONS}

In this section, we present some simple simulation results to evaluate the proposed single stage robust CRT algorithm and the two-stage robust CRT algorithm for integers with a general set of moduli. Let us first consider the case when $M_{1}=$ $9 \cdot 15, M_{2}=9 \cdot 20$ and $M_{3}=9 \cdot 18$. These three moduli do not satisfy the condition that $\Gamma_{i}, i=1,2,3$, are co-prime and thus the robust CRT obtained in [12], [13] can not be applied directly. However, we can use our proposed single stage robust CRT. According to Corollary 1, the maximal range of the determinable $N$ is 1620 and the maximal remainder error level $\tau$ for the robustness is upper bounded by $\tau<\frac{27}{4}$ from (27). In this simulation, the unknown integer $N$ is uniformly distributed in the interval $[0,1620)$. We consider the maximal remainder error levels $\tau=0,1,2,3,4,5,6$, and the errors are also uniformly distributed on $[0, \tau]$ in the remainders. 2000000 trials for each of them are implemented. The mean error $E(|\hat{N}-N|)$ between the estimated $\hat{N}$ in (4) and the true $N$ is plotted by the solid line marked with $\square$, and the theoretical estimation error upper bound in (6) is plotted by the solid line marked with $\triangle$ in Fig. 1. Obviously, one can see that for a general set of moduli the reconstruction errors of $N$ from the erroneous remainders are small compared to the range of $N$.

Next, we compare the robustness between the single stage and the two-stage robust CRT algorithms for the above same set of moduli. In this case, the conditions of the maximal remainder error levels for the single stage and the two-stage robust CRT algorithms of two groups $\left\{M_{1}, M_{2}\right\}$ and $\left\{M_{3}\right\}$ are $\frac{27}{4}$ and $\frac{45}{4}$, i.e., $\tau \leq 6$ and 11 , respectively. Let us consider the maximal remainder error levels $\tau$ from 0 to 25, and 2000000 trials for each of them. The unknown integer $N$ is taken as before. Fig. 2 shows the curves of the error bounds and the mean estimation errors $E(|\hat{N}-N|)$ for both the single stage and the two-stage robust CRT algorithms. Note that from our single stage robust CRT theory, the valid error bound for $\tau$ is only up to 6 , which 


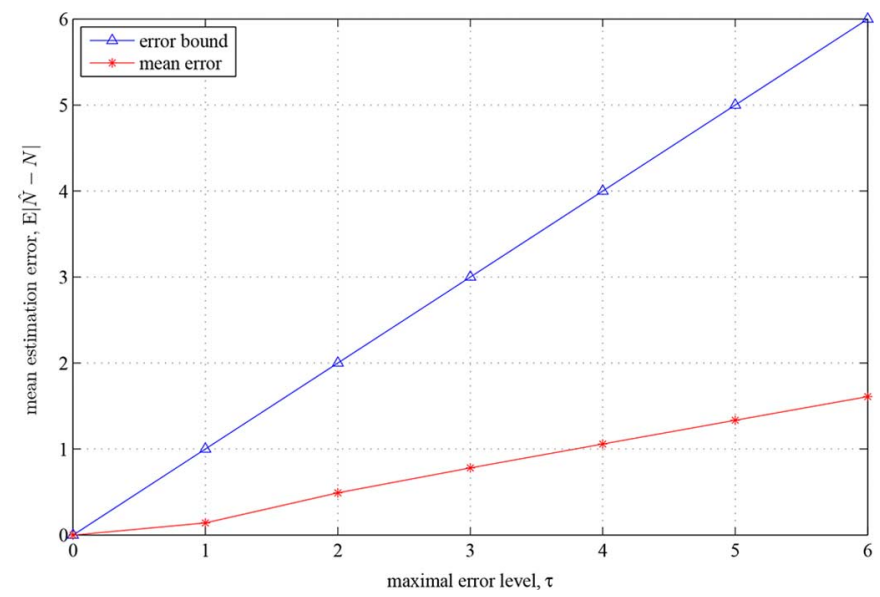

Fig. 1. Mean estimation error and theoretical error bound using the single stage robust CRT.

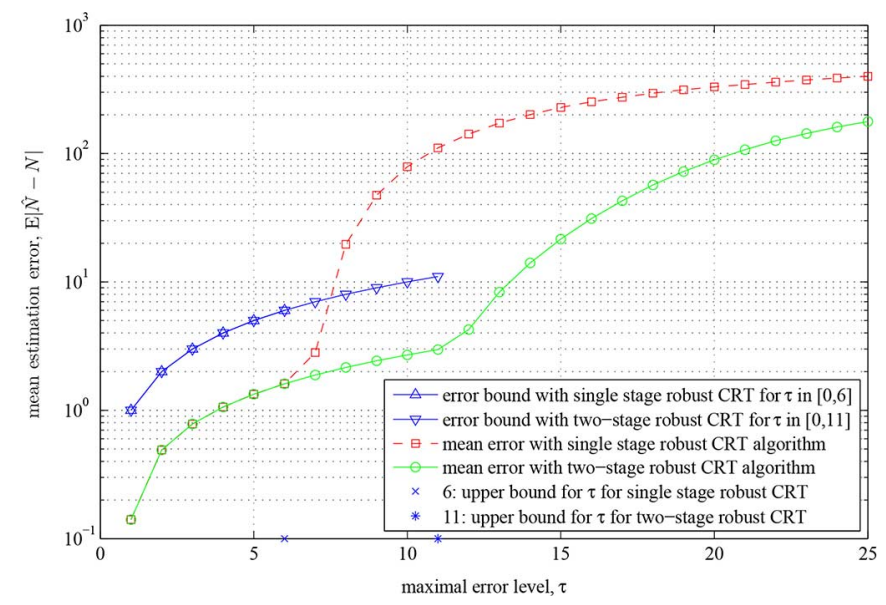

Fig. 2. Mean estimation error and theoretical error bound comparison using the single stage robust CRT and the two-stage robust CRT.

can be seen from the simulation results that the mean estimation error $E(|\hat{N}-N|)$ starts to deviate the previous line trend at $\tau=7$, then increases significantly and breaks the linear error bound when $\tau$ is further greater, i.e., robust reconstruction may not hold. On the other hand, with the two-stage robust CRT algorithm, one can see that the curve of the mean estimation error $E(|\hat{N}-N|)$ is always below the curve of the error bound, i.e., we can robustly reconstruct $N, 0 \leq N<1620$, even when the maximal error level is 11 that is the upper bound for $\tau$ obtained in this paper for the two-stage robust CRT algorithm. These simulation results confirm the theory obtained in this paper.

\section{CONCLUSION}

In this paper, we considered the robust reconstruction problem from erroneous remainders, namely robust CRT problem, for a general set of moduli that may not satisfy the condition needed in the previous robust CRT studies in [12], [13]. We obtained a necessary and sufficiency condition for the robust CRT when all the erroneous remainders are used together, called single stage robust CRT. Interestingly, our proposed single stage robust CRT may have better robustness than that of the robust CRT obtained in [12], [13] even when it could be applied. To further improve the robustness, we then proposed a multi-stage robust CRT, where the moduli are grouped into several groups. As an example, for the two-stage robust CRT, our proposed single stage robust CRT is first applied to each group and then applied across the groups second time. Also, an algorithm on how to group a given set of moduli was proposed. We finally presented some simulations to verify our proposed theory.

\section{REFERENCES}

[1] J. H. McClellan and C. M. Rader, Number Theory in Digital Signal Processing. Englewood Cliffs, NJ, USA: Prentice-Hall, 1979.

[2] C. Ding, D. Pei, and A. Salomaa, Chinese Remainder Theorem: Applications in Computing, Coding, Cryptography. Singapore: World Scientific, 1999

[3] O. Ore, "The general Chinese remainder theorem," Amer. Math. Month., vol. 59, no. 6, pp. 365-370, Jun.-Jul. 1952.

[4] Y. Wang, "New Chinese remainder theorems," in Proc. 32nd Asilomar Conf. Signals, Syst. Comput., Pacific Grove, CA, USA, Nov. 1998, pp. $165-171$.

[5] O. Goldreich, D. Ron, and M. Sudan, "Chinese remaindering with errors," IEEE Trans. Inf. Theory, vol. 46, no. 7, pp. 1330-1338, Jul. 2000.

[6] V. Guruswami, A. Sahai, and M. Sudan, "Soft-decision decoding of Chinese remainder codes," in Proc. 41st IEEE Symp. Found. Comput. Sci., Redondo Beach, CA, USA, 2000, pp. 159-168.

[7] I. E. Shparlinski and R. Steinfeld, "Noisy Chinese remaindering in the Lee norm," J. Complex., vol. 20, pp. 423-437, 2004.

[8] X.-G. Xia, "Estimation of multiple frequencies in undersampled complex valued waveforms," IEEE Trans. Signal Process., vol. 47, pp. 3417-3419, Dec. 1999.

[9] X.-G. Xia and K. Liu, "A generalized Chinese remainder theorem for residue sets with errors and its application in frequency determination from multiple sensors with low sampling rates," IEEE Signal Process. Lett., vol. 12, pp. 768-771, Nov. 2005.

[10] Z. Huang and Z. Wan, "Range ambiguity resolution in multiple PRF pulse Doppler radars," in Proc. Int. Conf. Acoust., Speech, Signal Process. (ICASSP), Dallas, TX, USA, Apr. 1987, pp. 1786-1789.

[11] X.-G. Xia and G. Wang, "Phase unwrapping and a robust Chinese remainder theorem," IEEE Signal Process. Lett., vol. 14, no. 4, pp. 247-250, Apr. 2007.

[12] X. W. Li, H. Liang, and X.-G. Xia, "A robust Chinese remainder theorem with its applications in frequency estimation from undersampled waveforms," IEEE Trans. Signal Process., vol. 57, no. 11, pp. 4314-4322, Nov. 2009.

[13] W.-J. Wang and X.-G. Xia, "A closed-form robust Chinese remainder theorem and its performance analysis," IEEE Trans. Signal Process., vol. 58, no. 11, pp. 5655-5666, Nov. 2010.

[14] W. Xu, E. C. Chang, L. K. Kwoh, H. Lim, and W. C. A. Heng, "Phase unwrapping of SAR interferogram with multi-frequency or multi-baseline," in Proc. IGARSS, 1994, pp. 730-732.

[15] D. P. Jorgensen, T. R. Shepherd, and A. S. Goldstein, "A dual-pulse repetition frequency scheme for mitigating velocity ambiguities of the NOAA P-3 airborne Doppler radar," J. Atmos. Ocean. Technol., vol. 17, no. 5, pp. 585-594, May 2000.

[16] G. Wang, X.-G. Xia, V. C. Chen, and R. L. Fiedler, "Detection, location, and imaging of fast moving targets using multifrequency antenna array SAR," IEEE Trans. Aerosp. Electron. Syst., vol. 40, no. 1, pp. 345-355, Jan. 2004.

[17] M. Ruegg, E. Meier, and D. Nuesch, "Capabilities of dual-frequency millimeter wave SAR with monopulse processing for ground moving target indication," IEEE Trans. Geosci. Remote Sens., vol. 45, no. 3, pp. 539-553, Mar. 2007.

[18] Y. M. Zhang and M. Amin, "MIMO radar exploiting narrowband frequency-hopping waveforms," in Proc. 16th Eur. Signal Process. Conf. (EUSIPCO 2008), Lausanne, Switzerland, Aug. 25-29, 2008.

[19] J. Bioucas-Dias, V. Katkovnik, J. Astola, and K. Egiazarian, "Multifrequency phase unwrapping from noisy data: Adaptive local maximum likelihood approach," Image Analysis, Lecture Notes in Comput. Sci., vol. 5575/2009, pp. 310-320, Jul. 2009. 
[20] W.-K. Qi, Y.-W. Dang, and W.-D. Yu, "Deblurring velocity ambiguity of distributed space-borne SAR based on Chinese remainder theorem," J. Electron. Inf. Tech., vol. 31, no. 10, pp. 2493-2496, Oct. 2009.

[21] G. Li, H. Meng, X.-G. Xia, and Y.-N. Peng, "Range and velocity estimation of moving targets using multiple stepped-frequency pulse trains," Sens., vol. 8, pp. 1343-1350, 2008.

[22] G. Li, J. Xu, Y.-N. Peng, and X.-G. Xia, "Location and imaging of moving targets using non-uniform linear antenna array," IEEE Trans. Aerosp. Electron. Syst., vol. 43, no. 3, pp. 1214-1220, Jul. 2007.

[23] X. W. Li and X.-G. Xia, "Location and imaging of elevated moving target using multi-frequency velocity SAR with cross-track interferometry," IEEE Trans. Aerosp. Electron. Syst., vol. 47, no. 2, pp. 1203-1212, Apr. 2011.

[24] B. Yang, W. J. Wang, X.-G. Xia, and Q. Yin, "Phase detection based range estimation with a dual-band robust Chinese remainder theorem," Sci. China-Inf. Sci., vol. 57, no. 2, pp. 1-9, Feb. 2014.

[25] W. C. Li, X. Z. Wang, X. M. Wang, and B. Moran, "Distance estimation using wrapped phase measurements in noise," IEEE Trans. Signal Process., vol. 61, no. 7, pp. 1676-1688, Apr. 2013.

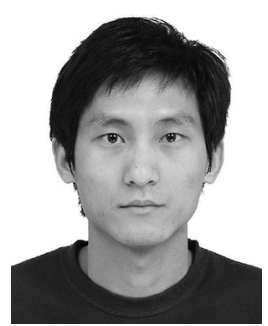

Li Xiao was born in Jiangxi, China. He received the B.S. degree in mathematics from Wuhan University, Wuhan, China, in 2009, and the M.S. degree in mathematics from Nankai University, Tianjin, China, in 2012.

He is currently working toward the Ph.D. degree in electrical engineering at Department of Electrical and Computer Engineering, University of Delaware, Newark. His research interests lie in the area of signal processing and communications.

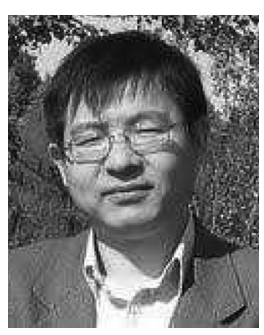

Xiang-Gen Xia (M'97-S'00-F'09) received the B.S. degree in mathematics from Nanjing Normal University, Nanjing, China, and the M.S. degree in mathematics from Nankai University, Tianjin, China, and the Ph.D. degree in electrical engineering from the University of Southern California, Los Angeles, in 1983, 1986, and 1992, respectively.

He was a Senior/Research Staff Member at Hughes Research Laboratories, Malibu, CA, during 1995-1996. In September 1996, he joined the Department of Electrical and Computer Engineering, University of Delaware, Newark, where he is the Charles Black Evans Professor. His current research interests include space-time coding, MIMO and OFDM systems, digital signal processing, and SAR and ISAR imaging. He has over 270 refereed journal articles published and accepted, and 7 U.S. patents awarded and is the author of the book Modulated Coding for Intersymbol Interference Channels (New York: Marcel Dekker, 2000).

Dr. Xia received the National Science Foundation (NSF) Faculty Early Career Development (CAREER) Program Award in 1997, the Office of Naval Research (ONR) Young Investigator Award in 1998, and the Outstanding Overseas Young Investigator Award from the National Nature Science Foundation of China in 2001. He also received the Outstanding Junior Faculty Award of the Engineering School of the University of Delaware in 2001. He is currently serving and has served as an Associate Editor for numerous international journals including the IEEE Transactions on Signal Processing, IEEE TRansactions on WIRELESS COMMUNICATIONS, IEEE TRANSACTIONS ON MOBILE COMPUTING, and IEEE Transactions on Vehicular Technology. He is Technical Program Chair of the Signal Processing Symposium, IEEE GLOBECOM 2007 in Washington DC, and the General Co-Chair of ICASSP 2005 in Philadelphia, PA.

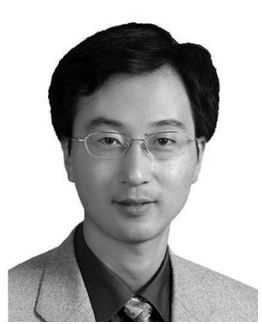

Wenjie Wang (M'10) received the B.S., M.S. and $\mathrm{Ph} . \mathrm{D}$. degrees in information and communication engineering from Xi'an Jiaotong University, Xi'an, China, in 1993, 1998, and 2001, respectively.

From 2009 to 2010, he was a visiting scholar at the Department of Electrical and Computer Engineering, University of Delaware, Newark. Currently, he is a Professor at Xi' an Jiaotong University. His main research interests include MIMO and OFDM systems, digital signal processing, and wireless sensor networks. 